\title{
The Sign-Definiteness Lemma and Its Applications to Robust Transceiver Optimization for Multiuser MIMO Systems
}

\author{
Ebrahim Avazkonandeh Gharavol and Erik G. Larsson
}

\section{Linköping University Post Print}

N.B.: When citing this work, cite the original article.

(C)2013 IEEE. Personal use of this material is permitted. However, permission to reprint/republish this material for advertising or promotional purposes or for creating new collective works for resale or redistribution to servers or lists, or to reuse any copyrighted component of this work in other works must be obtained from the IEEE.

Ebrahim Avazkonandeh Gharavol and Erik G. Larsson, The Sign-Definiteness Lemma and Its Applications to Robust Transceiver Optimization for Multiuser MIMO Systems, 2013, IEEE Transactions on Signal Processing, (61), 2, 238-252.

http://dx.doi.org/10.1109/TSP.2012.2222379

Postprint available at: Linköping University Electronic Press

http://urn.kb.se/resolve?urn=urn:nbn:se:liu:diva-85874 


\title{
The Sign-Definiteness Lemma and its
}

\section{Applications to Robust Transceiver}

\section{Optimization for Multiuser MIMO Systems}

\author{
Ebrahim A. Gharavol and Erik G. Larsson \\ Division of Communication Systems, Electrical Engineering Department (ISY) \\ Linköping University, 58183 Linköping, Sweden
}

\begin{abstract}
We formally generalize the sign-definiteness lemma to the case of complex-valued matrices and multiple norm-bounded uncertainties. This lemma has found many applications in the study of the stability of control systems, and in the design and optimization of robust transceivers in communications. We then present three different novel applications of this lemma in the area of multi-user multiple-input multiple-output (MIMO) robust transceiver optimization. Specifically, the scenarios of interest are: (i) robust linear beamforming in an interfering adhoc network, (ii) robust design of a general relay network, including the two-way relay channel as a special case, and (iii) a half-duplex one-way relay system with multiple relays. For these networks, we formulate the design problems of minimizing the (sum) MSE of the symbol detection subject to different average power budget constraints. We show that these design problems are non-convex (with bilinear or trilinear constraints) and semiinfinite in multiple independent uncertainty matrix-valued variables. We propose a two-stage solution where in the first step the semi-infinite constraints are converted to linear matrix inequalities using the generalized signdefiniteness lemma, and in the second step, we use an iterative algorithm based on alternating convex search (ACS). Via simulations we evaluate the performance of the proposed scheme.
\end{abstract}

\section{INTRODUCTION}

Robust design refers to a design problem in which there is some kind of uncertainty in the problem data or parameters. In control engineering, this is a well-established discipline and there are many standard texts on the subject, see, e.g., [1], [2] and the references therein. More recently, the robust design paradigm has been used for various applications in signal processing and communications, most notably antenna array beamforming problems. Some pioneering contributions for beamforming

Copyright (c) 2012 IEEE. Personal use of this material is permitted. However, permission to use this material for any other purposes must be obtained from the IEEE by sending a request to pubs-permissions@iee.org.

This paper describes work undertaken in the context of the LOLA project - Achieving LOw-LAtency in Wireless Communications (www.ict-lola.eu). The research leading to these results has received funding from the European Community 7 th Seventh Framework Program under grant agreement No. 248993. E. Larsson is a Royal Swedish Academy of Sciences (KVA) Research Fellow supported by a grant from the Knut and Alice Wallenberg Foundation. Parts of this paper were presented at the 49th Annual Allerton Conference 2011 [61], at the 45th Annual Asilomar Conference on Signals, Systems and Computers 2011 [62], and at the 4th IEEE International Workshop on Computational Advances in Multi-Sensor Adaptive Processing (CAMSAP) 2011 [63]. 
applications are contained in [3], [4], which introduced the concept of robust beamforming, taking uncertainties in the array response into account. Further fundamental contributions on robust beamforming can be found in, e.g., [5]-[13] (see also the references therein).

Many robust design problems can be cast as semi-infinite optimization problems (optimization problems with infinitely many constraints). The sign-definiteness lemma which was first proposed by I.A. Petersen [14] is a tool which reduces such a semi-infinite problem to a much simpler linear matrix inequality. This lemma was motivated by the study of stability of systems with uncertain parameters in control theory, and in that field it has also been used for robust $H_{\infty}$ control design. The lemma is also applicable to the worst-case transceiver optimization designs considered in this paper (which are completely different problems than $H_{\infty}$ design). Hence, the lemma as such is extremely versatile and useful in many different disciplines.

In this paper we generalize the sign-definiteness lemma to the case in which the system has multiple uncertainties and all variables are complex valued. As we will see, this generalization has many applications in joint robust transceiver optimization for multiuser multiple-input multiple-output (MIMO) communication systems. We will present three specific applications: (i) an interfering adhoc network, (ii) a general amplify-and-forward MIMO relay network (including two-way relaying as a special case), and (iii) a multi-relay network. However, the applications of the lemma are not limited to these three cases, and similar analysis and design strategies can be used for many related applications as well. For example, it is possible to extend our method to "cognitive radio" setups that involve additional constraints on the interference generated by the transmitters. The optimization variables in the applications that we consider are the precoder and equalization filters. Specifically, we design a set of linear precoders and equalizers, optimized under the assumption that only imperfect channel state information (CSI) is available. We model the CSI uncertainty using a ball-shaped norm bounded error (NBE) model and adopt a worst-case design methodology, resulting in a system design which maximizes performance for the worst possible CSI realization as defined by the NBE. We also extend the design problems to a cognitive radio (CR) setup. In this paper we use (sum) MSE to quantify performance, see Section II for more details.

The application setups that we consider here represent good models for many practically important scenarios. For example, the interference channel represented by the interfering adhoc network application is a common model for spectrum sharing in wireless networks [43]. The interference relay channel represented by our general relay network model can model, for example the scenario of a relay in a wireless system that is shared between multiple operators [45]. The two-way relay channel using amplify-and-forward processing is known to be an important building block both for future cellular and wireless sensor networks [44].

\section{A. Related Recent Work}

As already noted, robust optimization for beamforming applications has a significant history that started with the pioneering work of [3] and was followed by several early, important papers [4], [8], [10]. Later contributions to the topic include [5]-[7], [9], [11]-[13]. Next we will review the 
most recent works which are related to our applications. Related problems have been considered before for multiple-input single-output (MISO) and MIMO communication systems. For example in [15]-[19] the transmitters for the MISO broadcast channel (BC) were optimized using different design criteria including MSE, sum-MSE and signal-to-interference-plus-noise ratio (SINR) subject to different power constraints in different setups including CR networks. There are also many papers that robustly design similar systems with multiple antennas at both ends (i.e., the MIMO case) [20]-[25] in single-user or multi-user networks. All these problems are such that each semi-infinite constraint includes only one uncertainty variable, and they mostly resort to the complex-valued version of the sign-definiteness lemma published in [26] to resolve the semi-infiniteness of the constraints.

Similarly, there are several papers that study various aspects of the capacity and beamforming design for relay channels, especially for the two-way relay channel (TWRC) [27]-[35] and the interference relay channel (IRC) [36]-[42] with or without (CSI) uncertainty. In [36] the Gaussian IRC with different relaying schemes like compress-and-forward, compute-and-forward, and hash-and-forward is studied. The authors use a new approach to find an upper bound on the sum-rate capacity of such a system, by studying the strong and the weak interference regimes and establish the sum-capacity, which, in turn, serves as an upper bound on the sum-capacity of the Gaussian IRC with bounded relay power. The capacity of a cognitive relay-assisted Gaussian interference channel is studied in [37]. An achievable rate region for the system is derived by combining the Han-Kobayashi coding scheme for the general interference channel with dirty paper coding. Reference [37] also derives outer bounds on the capacity region and obtains the number of degrees of freedom of the system. A novel sum-rate outer bound for the Gaussian interference channel with a relay is presented in [38] and [39]. The power allocation problem for interference relay channels is considered in [40], [41]. Due to the competitive nature of the multiuser environment, the problem is modeled as a strategic non-cooperative game. It is shown that this game always has a unique Nash equilibrium. A hash-and-forward relaying scheme is studied in [42].

\section{B. Contributions}

This paper unifies the results presented in our conference papers [61]-[63] in a comprehensive form and presents additional discussion and comparisons. The specific contributions of the paper are:

- We generalize the sign-definiteness lemma to the case of complex-valued quantities with multiple uncertainties in each design constraint.

- We formulate and solve the linear beamforming problem in an interfering adhoc network. This contribution generalizes the method employed in [25] in which the authors assumed that each MSE constraint is only affected by one single uncertainty source.

- We propose a generalized model for a relaying network, which includes the cases of TWRC and IRC with direct links, and we optimally design precoders and equalizers for this network.

- We show that the theory developed for the aforementioned networks can be applied to a multirelay network as well. 
The philosophy of the presentation is to highlight the wide range of the applications of the signdefiniteness lemma, and to illuminate the similarities in the design methods used for the different network topologies. The structure of the remainder of this paper is as follows. System models and problem formulations for robust beamforming in the interfering adhoc network, in the general relay network, and in the multi-relay network are presented in Sections II-B, II-C and II-D, respectively. In Section III we present the generalized version of the sign-definiteness lemma and prove it. The robust solution to the previously introduced problems are given in Section IV. Simulation results are provided in Section V. Section VI concludes the paper.

Notation: Boldfaced letters are used for vectors or matrices. The field of the complex numbers, the field of $n$-dimensional complex vectors and the field of $m \times n$-dimensional complex-valued matrices are denoted by $\mathbb{C}, \mathbb{C}^{n}$ and $\mathbb{C}^{m \times n}$, respectively. For any vector $\boldsymbol{x}$ or for any matrix $\boldsymbol{X}$, $\|\boldsymbol{x}\|$ and $\|\boldsymbol{X}\|_{F}$ denote the Euclidean and the Frobenius norms of that vector or matrix, respectively. To denote the transpose and conjugate transpose of a matrix, $(\cdot)^{T}$ and $(\cdot)^{*}$ are used. $\Re\{\cdot\}$ denotes the real part of its argument. Positive semi-definiteness of a matrix $\boldsymbol{X}$ is denoted by $\boldsymbol{X} \succeq 0$. To show a vertically concatenated vectorized version of a matrix, we write vec [], and to show the block diagonal concatenation of a set of matrices we write blkdiag [...], respectively. Finally, $\mathrm{E}_{\boldsymbol{x}}[f(\boldsymbol{x})]$ is the expectation of $f(\boldsymbol{x})$ with respect to the stochastic variable $\boldsymbol{x}$.

\section{System Model ANd PRoblem Formulations}

In this section we present the system models and problem formulations of the three different applications. It should be noted that the application of the sign-definiteness lemma, which will be presented in the next sub-sections is not limited to these three networks. This lemma can be applied to any general robust mean-squared error estimation in the presence of model uncertainties (see, e.g., Section IV of [26]), especially when there are several independent uncertainties associated with each design constraint.

We will use an MSE based problem formulation. The MSE is a well established measure for the type of problem we consider [15]-[17], [19]-[25], [27] and in general in the context of severely delay constrained applications that use short codes or even uncoded transmission, see for example [54]-[56]. Note also that short inner codes optimized with respect to MSE may be used as building blocks in communication systems that have an outer error-correcting code, in a similar spirit to the way space-time block codes based on signal-space diversity techniques can be used as inner codes in coded multiantenna transmissions. ${ }^{1}$ Unfortunately, extending our framework to using achievable rate instead of MSE does not appear to be directly possible, and we have to leave this as an open problem. It is also noteworthy that throughout this paper we assume that the receivers, where applicable, treat the interference as noise and do not try to decode it first.

We will first give a brief background on the uncertainty and the model we use to describe it, and then we will proceed with the applications.

\footnotetext{
${ }^{1}$ Strictly speaking, using mutual information as performance metric is better than MSE in this context, e.g., see [57].
} 


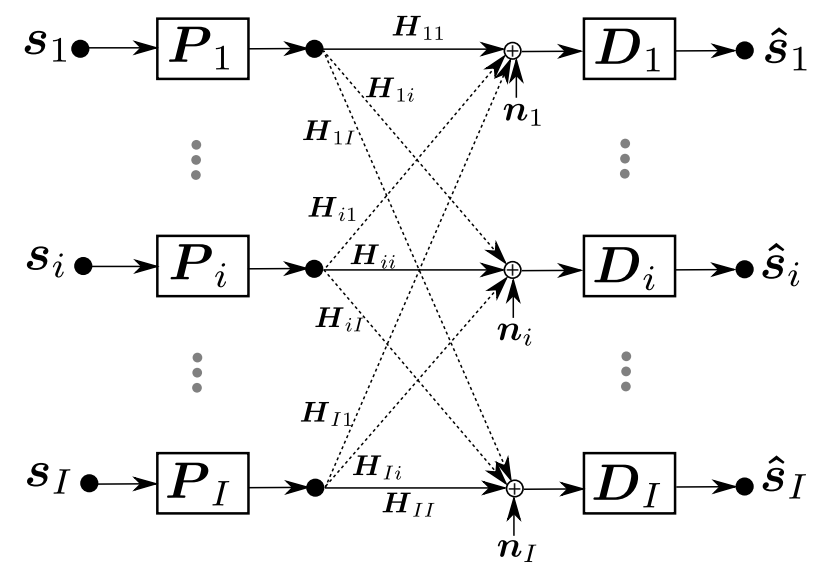

Fig. 1. Signal flow graph of an interfering adhoc network

\section{A. Background}

In the following examples, we assume that the model uncertainty is due to uncertainty in the CSI. Since we are interested in MIMO communications, an uncertain CSI, say $\boldsymbol{H} \in \mathbb{C}^{m \times n}$ can be described using the following NBE model (see, e.g., [4] and the references therein):

$$
\boldsymbol{H} \in \mathcal{H} \triangleq\left\{\tilde{\boldsymbol{H}}+\boldsymbol{\Delta} \mid\|\boldsymbol{\Delta}\|_{F} \leq \delta\right\}
$$

In this notation $\tilde{\boldsymbol{H}}$ is the nominal value of the channel (that is known to the transmitter and receiver) and $\boldsymbol{\Delta}$ is the deterministically unknown perturbation term of $\boldsymbol{H}$. The only information that we have regarding $\Delta$ is that it is norm bounded.

The motivation to use a spherical uncertainty region is twofold: (1) with Gaussian distributions, this is the region with the smallest volume that contains a given probability mass. (2) Given knowledge only of the error magnitude range but no other information about it, a spherical uncertainty region is reasonable from a symmetry point of view.

In today's communication systems, the receiver typically estimates the CSI using pilot symbols sent from the transmitter side. The receiver usually sends this CSI back to the transmitter side. Because of estimation errors, the CSI is generally uncertain, and owing to quantization errors the uncertainty of the CSI at the transmitter could be different from the uncertainty of the CSI at the receiver. In this paper, however, we use a single model to describe both uncertainties. This is simple, and moreover it is natural if the design of both the transmitter and receiver filters is done jointly at a central processing node, and then both the transmitter and the receiver are informed of the matrices that they should use. In this case, there is only nominal version of each channel, although the receivers could have done somewhat better as they would typically have a higher quality CSI.

\section{B. Application 1: Multiuser Interfering Network}

1) System Model: The first application is transceiver optimization for an interfering adhoc network, see Fig. 1. In this network, $I$ independent links (Tx-Rx pairs) are communicating over a shared channel. In the $i$ th link, $i=1, \cdots, I$; the Tx node aims to send $S_{i}$ independent data streams using 
$T_{i}$ antennas towards the destination ( $\mathrm{Rx}$ node) which is equipped with $R_{i}$ receive antennas. We assume that the symbols emitted by the source are zero-mean, independent and have equal energy, i.e., $\mathrm{E}_{\boldsymbol{s}_{i}}\left[\boldsymbol{s}_{i}\right]=0, \mathrm{E}_{\boldsymbol{s}_{i}}\left[\boldsymbol{s}_{i} \boldsymbol{s}_{i}^{*}\right]=\boldsymbol{I}$, where $\boldsymbol{s}_{i} \in \mathbb{C}^{S_{i}}$ is the transmitted vector of the source node. This information is linearly precoded using the precoding matrix $\boldsymbol{P}_{i} \in \mathbb{C}^{T_{i} \times S_{i}}$ and is sent towards the destination over a Rayleigh fading wireless MIMO channel $\boldsymbol{H}_{i i} \in \mathbb{C}^{R_{i} \times T_{i}}$. Each link also interferes with the other links and the unintended receivers receive the transmitted signal over the channels $\boldsymbol{H}_{i j} \in \mathbb{C}^{R_{j} \times T_{i}}, j=1, \cdots, I$, and $j \neq i$. In each Rx node, the received data is linearly equalized using the receive matrix $\boldsymbol{D}_{i} \in \mathbb{C}^{S_{i} \times R_{i}}$. The received vector signal at the receiver is equal to

$$
\hat{\boldsymbol{s}}_{i}=\boldsymbol{D}_{i}\left(\boldsymbol{H}_{i i} \boldsymbol{P}_{i} \boldsymbol{s}_{i}+\sum_{\substack{j=1 \\ j \neq i}}^{I} \boldsymbol{H}_{j i} \boldsymbol{P}_{j} \boldsymbol{s}_{j}+\boldsymbol{n}_{i}\right)
$$

where $\boldsymbol{n}_{i} \in \mathbb{C}^{R_{i}}$ is zero-mean white Gaussian noise with variance $\sigma_{i}^{2}$.

Unlike in conventional design problems, in this paper, the CSI is not assumed to be perfectly known. We use a NBE model framework [4] to describe the imperfect CSI. More precisely, $\boldsymbol{H}_{i j}$ can take on values as follows:

$$
\boldsymbol{H}_{i j} \in \mathcal{H}_{i j}=\left\{\tilde{\boldsymbol{H}}_{i j}+\boldsymbol{\Delta}_{i j} \mid\left\|\boldsymbol{\Delta}_{i j}\right\|_{F} \leq \delta_{i j}\right\}
$$

where $\tilde{\boldsymbol{H}}_{i j}$ is the nominal CSI and $\boldsymbol{\Delta}_{i j}$ denotes the norm-bounded uncertainty. To best design this network, we can jointly optimize the precoding and equalization matrices in different ways as will be described in the following sections.

2) Worst-case Problem Formulation: In the problem formulations that we target in this paper, the MSE of each link $\left(\mathrm{MSE}_{i}, \forall i\right)$ and the transmit power of each Tx node $\left(\operatorname{TxP}_{i}, \forall i\right)$ play important roles. These quantities are defined as follows:

$$
\begin{aligned}
\operatorname{TxP}_{i} & \triangleq \mathrm{E}_{\boldsymbol{s}_{i}}\left[\left\|\boldsymbol{P}_{i} \boldsymbol{s}_{i}\right\|^{2}\right], \forall i, \\
\mathrm{MSE}_{i} & \triangleq \mathrm{E}_{\left\{\boldsymbol{s}_{i}\right\}_{\forall i}}\left[\left\|\hat{\boldsymbol{s}}_{i}-\boldsymbol{s}_{i}\right\|^{2}\right], \quad \forall i, \\
& =\mathrm{E}_{\left\{\boldsymbol{s}_{i}\right\}_{\forall i}}\left[\left\|\left(\boldsymbol{D}_{i} \boldsymbol{H}_{i i} \boldsymbol{P}_{i}-\boldsymbol{I}_{S_{i}}\right) \boldsymbol{s}_{i}+\sum_{\substack{j=1 \\
j \neq i}}^{I} \boldsymbol{D}_{i} \boldsymbol{H}_{j i} \boldsymbol{P}_{j} \boldsymbol{s}_{j}+\boldsymbol{D}_{i} \boldsymbol{n}_{i}\right\|^{2}\right], \quad \forall i .
\end{aligned}
$$

We will give the exact expressions for these quantities below. In the following problem formulations we exploit the worst-case methodology, i.e., the constraints of the resulting optimization problems should be satisfied for all possible channel realizations defined based on the NBE framework of (3), including the least favorable one. It is clear that the least favorable (worst-case) channel realization results in the largest MSE or transmit power.

One way to formulate the design problem for this network is to minimize the system-wide sum MSE of the symbol detection subject to an average transmit power constraint for each link. This 
optimization problem in its epigraph form can be written as follows:

$$
\begin{aligned}
\underset{\left\{\boldsymbol{P}_{i}, \boldsymbol{D}_{i}, \tau_{i} \geq 0\right\}_{i=1}^{I}}{\operatorname{minimize}} & \sum_{i=1}^{I} \tau_{i} \\
\text { subject to } & \operatorname{TxP}_{i} \leq \gamma_{i}, \quad \forall i, \\
& \operatorname{MSE}_{i} \leq \tau_{i}, \quad\left\{\forall \boldsymbol{H}_{i j} \in \mathcal{H}_{i j}\right\}_{i, j}, \forall i .
\end{aligned}
$$

where $\gamma_{i}$ is the power budget for the $i$ th link. An alternative way of formulating the problem is to minimize the sum-transmit power, subject to MSE constraints $\left(\mu_{i}\right)$, as follows:

$$
\begin{aligned}
\underset{\left\{\boldsymbol{P}_{i}, \boldsymbol{D}_{i}, \tau_{i} \geq 0\right\}_{i=1}^{I}}{\operatorname{minimize}} & \sum_{i=1}^{I} \tau_{i} \\
\text { subject to } & \operatorname{TxP}_{i} \leq \tau_{i}, \quad \forall i, \\
& \operatorname{MSE}_{i} \leq \mu_{i}, \quad\left\{\forall \boldsymbol{H}_{i j} \in \mathcal{H}_{i j}\right\}_{i, j}, \forall i .
\end{aligned}
$$

Although both these formulations are useful from the perspective of system design, none of them will guarantee fairness among the different links. In the designs mentioned above, it is possible that the link with the best CSI is allocated most of the available resources of the network, which leads to poor service for the others. To prevent this unwanted event, we propose the following min-max fairness formulation, in which the network is optimized to guarantee the best possible service for the weakest link. This min-max fairness problem formulation for the MSE minimization can be written as follows:

$$
\begin{array}{cl}
\underset{\left\{\boldsymbol{P}_{i}, \boldsymbol{D}_{i}\right\}}{\operatorname{minimize}} & \max _{i j} \in \mathcal{H}_{i j}, \forall i, j \\
\text { subject to } & \operatorname{TxP}_{i} \leq \gamma_{i} .
\end{array}
$$

which is equivalent to the following problem:

$$
\begin{aligned}
\underset{\left\{\boldsymbol{P}_{i}, \boldsymbol{D}_{i}\right\}_{i=1}^{I}, \tau \geq 0}{\operatorname{minimize}} & \tau \\
\text { subject to } & \operatorname{TxP}_{i} \leq \gamma_{i}, \quad \forall i, \\
& \operatorname{MSE}_{i} \leq \tau \quad\left\{\forall \boldsymbol{H}_{i j} \in \mathcal{H}_{i j}\right\}_{i, j}, \forall i .
\end{aligned}
$$

It is clear that despite the different perspectives based on which the optimization problem is formulated, the resulting optimization problems have a similar structure, and it suffices to give solution details for one of them. By appropriately introducing slack variables, a similar procedure can be applied to other formulations.

\section{Application 2: General Non-Regenerative Amplify and Forward Interfering Relay Network}

1) System Model: The signal flow diagram of a general wireless relay system is depicted in Fig. 2. In this system, two distinct transmitter-receiver pairs simultaneously communicate with each other in two consecutive time slots. Each pair has its own transmit and receive sides. Unlike the one-way relay channel in which, to initiate a half-duplex communication service, two distinct time slots are 


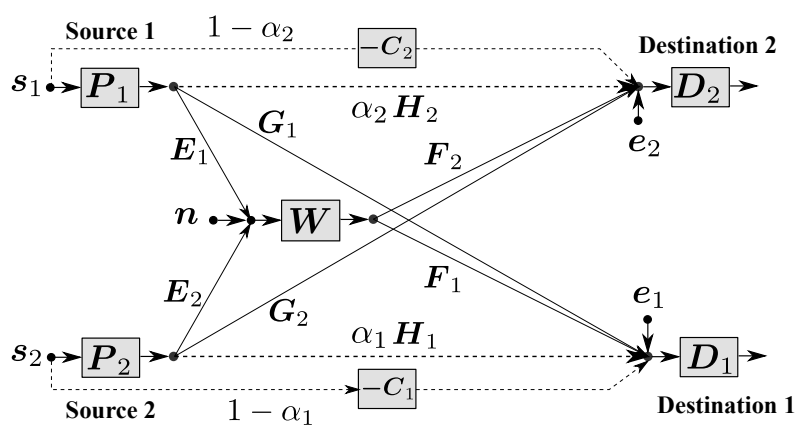

Fig. 2. Signal Flow Graph of a General Relay Channel

required, in this system two-way communication is provided using two time slots. In the first time slot, both terminals simultaneously transmit their data to the destination and to the relay node. In the second time slot, the relay node broadcasts a combination of the signal that it received in the first time slot, after performing a linear processing (beamforming) operation. Both other receivers listen during the second time slot. By means of this relaying process, exchange of the data is facilitated between the two transmitters and the two receivers. Effectively, the relaying operation serves to increase the SINR.

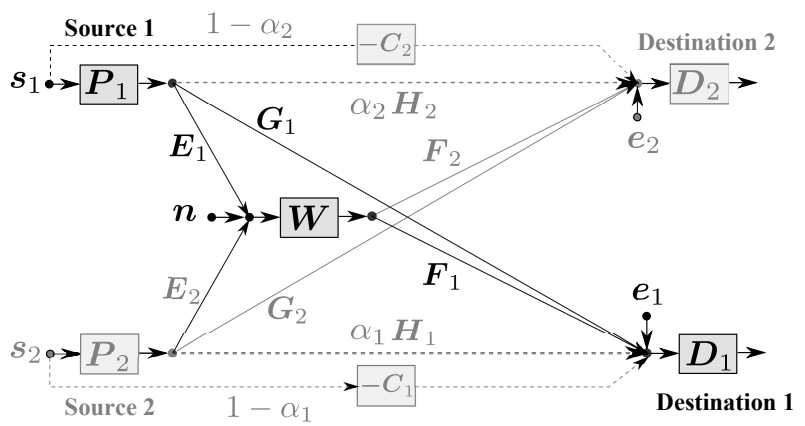

Fig. 3. Signal flow graph of a one-way relay channel

This model is general enough to cover one-way relay channels, two-way relay channels, and Gaussian interfering relay channels. If only one link exists in this system (see Fig. 3), i.e., $\boldsymbol{s}_{1}, \boldsymbol{F}_{1}, \boldsymbol{G}_{1}$, and $\boldsymbol{E}_{1}$ are the only non-zero elements, the system model reduces to that of a one-way relay channel. If $\boldsymbol{G}_{1}$ is zero, we have a one-way relay channel without a direct link. If $\boldsymbol{H}_{2}$ is non-zero, we have a model for a relay channel in a cognitive radio setup. The second receiver can be seen as a primary user to be protected. If two links simultaneously exist, but the destination nodes are cross co-located, then by choosing $\alpha_{1}=\alpha_{2}=0$ the self-cancellation filters will be activated, and we have a model for the two-way relay channel. In the more general case of two interfering links with no self cancellation, i.e., $\alpha_{1}=\alpha_{2}=1$, this model describes a Gaussian interfering network.

To transmit $S_{i}, i=1,2$, independent streams of zero-mean, unit-variance data $\left(s_{i} \in \mathbb{C}^{S_{i}}, i=\right.$ $1,2)$ with independent elements towards either end of the communication link, each transmitter uses $T_{i}, i=1,2$, transmit antennas. The receivers are equipped with $R_{i}, i=1,2$, receive antennas, while 
the relay has $r$ and $t$ receive and transmit antennas, respectively. The source nodes precode the data using the precoding matrices $\boldsymbol{P}_{i} \in \mathbb{C}^{T_{i} \times S_{i}}$. The precoded data is then sent over the wireless fading channels $\boldsymbol{G}_{i} \in \mathbb{C}^{R_{i} \times T_{i}}, \boldsymbol{H}_{i} \in \mathbb{C}^{R_{i} \times T_{-i}}, \boldsymbol{E}_{i} \in \mathbb{C}^{r \times T_{i}}, i=1,2$, where $\boldsymbol{X}_{-i}$ with the subscript $-i$ refers to a quantity belonging to the other index in the bi-index notation we use here. That is

$$
\boldsymbol{X}_{-i}= \begin{cases}\boldsymbol{X}_{2}, & i=1, \\ \boldsymbol{X}_{1}, & i=2 .\end{cases}
$$

At the relay node, the received signal is amplified using $W \in \mathbb{C}^{t \times r}$. The resulting signal is transmitted to the destinations in the next time slot over the channels $\boldsymbol{F}_{i} \in \mathbb{C}^{R_{i} \times t}, i=1,2$.

In the case of a TWRC, we set $\alpha_{1}=\alpha_{2}=0$, since each terminal knows its own transmitted signal. Then, first a self-cancellation filter, $-C_{i} \in \mathbb{C}^{R_{i} \times S_{-i}}$, is applied to the received signal. The aim of this filter is to suppress each node's own signal which has been retransmitted by the relay. After appropriately combining the received signals and decoding using the linear equalizers $\boldsymbol{D}_{i} \in$ $\mathbb{C}^{S_{i} \times R_{i}}, i=1,2$, the received signals are as follows:

$$
\begin{aligned}
\hat{\boldsymbol{s}}_{i}= & \boldsymbol{D}_{i}\left(\boldsymbol{G}_{i}+\boldsymbol{F}_{i} \boldsymbol{W} \boldsymbol{E}_{i}\right) \boldsymbol{P}_{i} \boldsymbol{s}_{i}+\boldsymbol{D}_{i}\left[\left(\alpha_{i} \boldsymbol{H}_{i}+\boldsymbol{F}_{i} \boldsymbol{W} \boldsymbol{E}_{-i}\right) \boldsymbol{P}_{-i}-\left(1-\alpha_{i}\right) \boldsymbol{C}_{i}\right] \boldsymbol{s}_{-i} \\
& +\boldsymbol{D}_{i} \boldsymbol{F}_{i} \boldsymbol{W} \boldsymbol{n}+\boldsymbol{D}_{i} \boldsymbol{e}_{i} .
\end{aligned}
$$

In this equation $\boldsymbol{n} \in \mathbb{C}^{r}$ and $\boldsymbol{e}_{i} \in \mathbb{C}^{R_{i}}$ are additive zero-mean noise signals with independent elements and variances $\sigma_{u}^{2}$ and $\sigma_{e_{i}}^{2}$, respectively. Due to the limited feedback between the nodes, it is assumed that only the nominal value of the CSI is known to the system and that the CSI follows the norm bounded error model. That is, for any complex-valued matrix quantity such as $\boldsymbol{E}_{i,-i}, \boldsymbol{F}_{i,-i}, \boldsymbol{G}_{i,-i}, \boldsymbol{H}_{i,-i}$, say $\boldsymbol{X}$, we have:

$$
\boldsymbol{X} \in \mathcal{S}_{\boldsymbol{X}}=\left\{\tilde{\boldsymbol{X}}+\boldsymbol{\Delta}_{\boldsymbol{X}} \mid\left\|\boldsymbol{\Delta}_{\boldsymbol{X}}\right\|_{F} \leq \delta_{\boldsymbol{X}}\right\}
$$

where $\tilde{\boldsymbol{X}}$ is the fixed nominal value of the CSI for each of the channels and $\boldsymbol{\Delta}_{\boldsymbol{X}}$ is the normbounded variation (uncertainty) around this nominal value. We next use this signal model to formulate optimization problems.

2) Problem Formulation: Our goal is to jointly optimize the source precoders, the relay beamformer, and the destination equalizers. To do so, we can formulate our design problem either in terms of a min-max fairness, relay transmit power minimization, or system-wide sum-MSE (SMSE) minimization. After choosing the performance measure of the system, we can consider the corresponding optimization problem with the power budgets of both sources and the relay node, or the allowed MSE of each link.

To facilitate the computation of the MSE of each link, and the transmit powers of the sources and the relay nodes, we use Lemma 2 presented in Section IV. Based on this lemma, the MSE of the links $\left(\mathrm{MSE}_{i}, i=1,2\right)$, and the transmit powers of the source $\left(\operatorname{TxP}_{s_{i}}, i=1,2\right)$ and the relay $\left(\operatorname{TxP}_{r}\right)$ 
nodes are given as follows:

$$
\begin{aligned}
\operatorname{TxP}_{s_{i}} \triangleq & \mathrm{E}_{\boldsymbol{s}_{i}}\left[\left\|\boldsymbol{P}_{i} \boldsymbol{s}_{i}\right\|_{2}^{2}\right], \quad i=1,2 \\
= & \left\|\boldsymbol{P}_{i}\right\|_{F}^{2}, \\
\mathrm{TxP}_{r} \triangleq & \mathrm{E}_{\boldsymbol{s}_{1,2}, \boldsymbol{u}}\left[\left\|\boldsymbol{W}\left(\boldsymbol{E}_{1} \boldsymbol{P}_{1} \boldsymbol{s}_{1}+\boldsymbol{E}_{2} \boldsymbol{P}_{2} \boldsymbol{s}_{2}\right)+\boldsymbol{W} \boldsymbol{u}\right\|_{2}^{2}\right] \\
= & \left\|\boldsymbol{W} \boldsymbol{E}_{1} \boldsymbol{P}_{1}\right\|_{F}^{2}+\left\|\boldsymbol{W} \boldsymbol{E}_{2} \boldsymbol{P}_{2}\right\|_{F}^{2}+\sigma_{u}^{2}\|\boldsymbol{W}\|_{F}^{2}, \\
\mathrm{MSE}_{i} \triangleq & \mathrm{E}_{\boldsymbol{s}_{1,2}, \boldsymbol{u}, \boldsymbol{e}_{1,2}}\left[\left\|\hat{\boldsymbol{s}}_{i}-\boldsymbol{s}_{i}\right\|^{2}\right], \quad i=1,2 \\
= & \left\|\boldsymbol{D}_{i}\left(\boldsymbol{G}_{i}+\boldsymbol{F}_{i} \boldsymbol{W} \boldsymbol{E}_{i}\right) \boldsymbol{P}_{i}-\boldsymbol{I}\right\|_{F}^{2}+\sigma_{u}^{2}\left\|\boldsymbol{D}_{i} \boldsymbol{F}_{i} \boldsymbol{W}\right\|_{F}^{2} \\
& +\left\|\boldsymbol{D}_{i}\left[\left(\alpha_{i} \boldsymbol{H}_{i}+\boldsymbol{F}_{i} \boldsymbol{W} \boldsymbol{E}_{-i}\right) \boldsymbol{P}_{-i}-\left(1-\alpha_{i}\right) \boldsymbol{C}_{i}\right]\right\|_{F}^{2}+\sigma_{e_{i}}^{2}\left\|\boldsymbol{D}_{i}\right\|_{F}^{2}
\end{aligned}
$$

Using these quantities, the problem formulation in its epigraph form to minimize the SMSE is as follows:

$$
\begin{aligned}
\underset{\boldsymbol{P}_{i}, \boldsymbol{W}, \boldsymbol{D}_{i}, \boldsymbol{C}_{i}, \tau_{i} \geq 0}{\operatorname{minimize}} & \tau_{1}+\tau_{2} \\
\text { subject to } & \operatorname{TxP}_{s_{i}} \leq P_{s_{i}}, i=1,2, \\
& \operatorname{TxP}_{r} \leq P_{r}, \forall\left(\boldsymbol{E}_{i}, \boldsymbol{E}_{-i}\right) \in \mathcal{S}_{\boldsymbol{E}_{i}} \times \mathcal{S}_{\boldsymbol{E}_{-i}}, i=1,2, \\
& \operatorname{MSE}_{i} \leq \tau_{i}, \forall \boldsymbol{E}_{i} \in \mathcal{S}_{\boldsymbol{E}_{i}}, \boldsymbol{E}_{-i} \in \mathcal{S}_{\boldsymbol{E}_{-i}}, \boldsymbol{F}_{i} \in \mathcal{S}_{\boldsymbol{F}_{i}}, \boldsymbol{G}_{i} \in \mathcal{S}_{\boldsymbol{G}_{i}}, \boldsymbol{H}_{i} \in \mathcal{S}_{\boldsymbol{H}_{i}}, i=1,2,
\end{aligned}
$$

where $P_{s_{i}}$ and $P_{r}$ are the power budgets of the source nodes and the relay node. The design problem to minimize the transmit power of the relay, and the min-max fairness problem are as follows, respectively:

$\underset{\boldsymbol{P}_{i}, \boldsymbol{W}, \boldsymbol{D}_{i}, \boldsymbol{C}_{i}, \tau \geq 0}{\operatorname{minimize}} \tau$

$$
\begin{array}{ll}
\text { subject to } & \operatorname{TxP}_{s_{i}} \leq P_{s_{i}}, i=1,2, \\
& \operatorname{TxP}_{r} \leq \tau, \forall \boldsymbol{E}_{i} \in \mathcal{S}_{\boldsymbol{E}_{i}}, \boldsymbol{E}_{-i} \in \mathcal{S}_{\boldsymbol{E}_{-i}}, i=1,2 \\
& \operatorname{MSE}_{i} \leq \gamma_{i}, \forall \boldsymbol{E}_{i} \in \mathcal{S}_{\boldsymbol{E}_{i}}, \boldsymbol{E}_{-i} \in \mathcal{S}_{\boldsymbol{E}_{-i}}, \boldsymbol{F}_{i} \in \mathcal{S}_{\boldsymbol{F}_{i}}, \boldsymbol{G}_{i} \in \mathcal{S}_{\boldsymbol{G}_{i}}, \boldsymbol{H}_{i} \in \mathcal{S}_{\boldsymbol{H}_{i}}, i=1,2,
\end{array}
$$

and

$$
\begin{aligned}
\underset{\boldsymbol{P}_{i}, \boldsymbol{W}, \boldsymbol{D}_{i}, \boldsymbol{C}_{i}, \tau \geq 0}{\operatorname{minimize}} & \tau \\
\text { subject to } & \mathrm{TxP}_{s_{i}} \leq P_{s_{i}}, i=1,2, \\
& \operatorname{TxP}_{r} \leq P_{r}, \forall \boldsymbol{E}_{i} \in \mathcal{S}_{\boldsymbol{E}_{i}}, \boldsymbol{E}_{-i} \in \mathcal{S}_{\boldsymbol{E}_{-i}}, i=1,2, \\
& \operatorname{MSE}_{i} \leq \tau, \forall \boldsymbol{E}_{i} \in \mathcal{S}_{\boldsymbol{E}_{i}}, \boldsymbol{E}_{-i} \in \mathcal{S}_{\boldsymbol{E}_{-i}}, \boldsymbol{F}_{i} \in \mathcal{S}_{\boldsymbol{F}_{i}}, \boldsymbol{G}_{i} \in \mathcal{S}_{\boldsymbol{G}_{i}}, \boldsymbol{H}_{i} \in \mathcal{S}_{\boldsymbol{H}_{i}}, i=1,2,
\end{aligned}
$$

where $P_{s_{i}}$ and $P_{r}$ are the power limits of the source and the relay nodes, and $\gamma_{i}, i=1,2$ are the MSE targets for each link. All these three variants of the design problem have a similar structure, and the theory which underlies each of them can be applied to the other two. Therefore, in Section IV we only give further details on the solution to the SMSE problem formulation. 


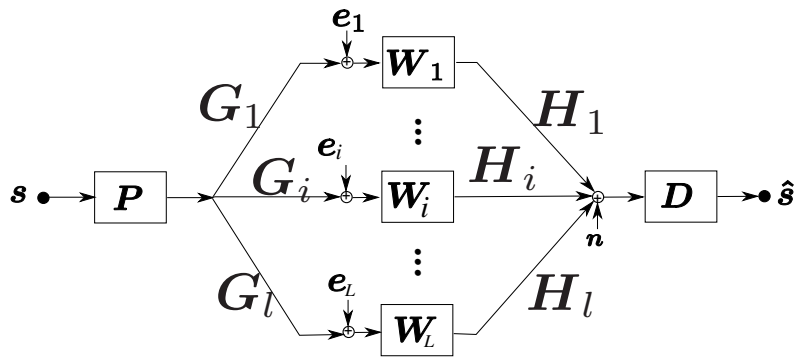

Fig. 4. Signal flow graph of a general relay channel with multiple relays

\section{Application 3: Multi-Relay Network}

1) System Model: Fig. 4 depicts the multiple-relay network application. There is a source node with $T$ transmit antennas which is transmitting $S$ streams of zero-mean, unit-variance independent information symbols, i.e., $s \in \mathbb{C}^{S}$, to the destination. The destination has $R$ receive antennas. In this system $L$ independent relay nodes help the source. Each relay node is equipped with $r_{i}, i=1, \cdots, L$ receive and $t_{i}, i=1, \cdots, L$, transmit antennas. The CSI between the source and the relay is represented by $\boldsymbol{G}_{i} \in \mathbb{C}^{r_{i} \times T}$ and the CSI between the relay nodes and the destination is represented by $\boldsymbol{H}_{i} \in \mathbb{C}^{R \times t_{i}}$. We will use linear precoding, equalizing and beamforming at the source, destination and relay nodes, defined by the linear operators (matrices) $\boldsymbol{P} \in \mathbb{C}^{T \times S}, \boldsymbol{D} \in \mathbb{C}^{S \times R}$ and $\boldsymbol{W}_{i} \in \mathbb{C}^{t_{i} \times r_{i}}$. We assume that the noise affecting the received signals at the relay and the destination is zero-mean and Gaussian.

The received signal at the destination is

$$
\hat{\boldsymbol{s}}=\boldsymbol{D}\left(\sum_{i=1}^{L} \boldsymbol{H}_{i} \boldsymbol{W}_{i} \boldsymbol{G}_{i} \boldsymbol{P} \boldsymbol{s}+\sum_{i=1}^{L} \boldsymbol{H}_{i} \boldsymbol{W}_{i} \boldsymbol{e}_{i}+\boldsymbol{n}\right)
$$

The noise terms $\boldsymbol{e}_{i} \in \mathbb{C}^{r_{i}}$ and $\boldsymbol{n} \in \mathbb{C}^{R}$ are zero-mean Gaussian with variances $\sigma_{e_{i}}^{2}$ and $\sigma_{n}^{2}$, respectively. As before we assume that the CSI belongs to a norm bounded uncertainty region:

$$
\begin{aligned}
\boldsymbol{H}_{i} \in \mathcal{H}_{i} & =\left\{\tilde{\boldsymbol{H}}_{i}+\boldsymbol{\Delta}_{i} \mid\left\|\boldsymbol{\Delta}_{i}\right\|_{F} \leq \delta_{i}\right\}, \\
\boldsymbol{G}_{i} \in \mathcal{G}_{i} & =\left\{\tilde{\boldsymbol{G}}_{i}+\boldsymbol{\Lambda}_{i} \mid\left\|\boldsymbol{\Lambda}_{i}\right\|_{F} \leq \lambda_{i}\right\} .
\end{aligned}
$$

2) Worst-case Problem Formulation: We next formulate the design problem which is based on minimizing the system-wide MSE (MSE) of the symbol detection subject to power constraints at the source $\left(\mathrm{TxP}_{s}\right)$ and relay nodes $\left(\operatorname{TxP}_{r_{i}}\right)$. The problem in its epigraph form is

$$
\begin{aligned}
\underset{\boldsymbol{P}, \boldsymbol{D},\left\{\boldsymbol{W}_{i}\right\}_{i}, \tau \geq 0}{\operatorname{minimize}} & \tau \\
\text { subject to } & \operatorname{TxP}_{s} \leq P_{s}, \\
& \operatorname{TxP}_{r_{i}} \leq P_{r_{i}}, \quad \forall \boldsymbol{G}_{i} \in \mathcal{G}_{i}, \forall i, \\
& \operatorname{MSE} \leq \tau, \quad\left\{\forall \boldsymbol{G}_{i} \in \mathcal{G}_{i}\right\}_{i},\left\{\forall \boldsymbol{H}_{i} \in \mathcal{H}_{i}\right\}_{i}, \forall i,
\end{aligned}
$$

where $P_{s}$ and $P_{r_{i}}$ are the power budgets for the source and the relay nodes. It is possible to formulate the design problem using different objectives, such as sum-power minimization or minimization of 
the maximum relay transmit power. However, all these different formulations have a similar structure, and we only focus on the above problem.

\section{E. Remarks about the Problem Formulations}

Referring back to the problem formulations given above, the following remarks can be made:

Remark 1: It is clear that $\mathrm{TxP}_{s_{i}}, i=1,2, \mathrm{TxP}_{r}$, and $\mathrm{MSE}_{i}, i=1,2$, are linear, bilinear, and trilinear functions of the design variables, respectively. Hence, $\operatorname{TxP}_{r}$, and $\mathrm{MSE}_{i}, i=1,2$, are non-convex in the design variables.

Remark 2: In these robust problem formulations, the last constraints are semi-infinite, i.e., they represent an infinite number of constraints. Moreover, the constraints are not simultaneously convex in the design variables. Both these properties make the problem very hard to solve (it is NP-hard ${ }^{2}$ ).

Remark 3: Clearly if we set $\delta_{\boldsymbol{X}}=0, \mathcal{S}_{\boldsymbol{X}}$ becomes a singleton set, comprising only the nominal value of the CSI for each channel. This case is the perfect CSI scenario and we will use this scenario as a benchmark when assessing the performance of the algorithm proposed here.

In the following sections we first present a lemma to deal with semi-infinite constraints, and then we propose a two-stage mechanism to simplify each problem into an equivalent problem. We then provide an iterative algorithm which solves the equivalent problem near-optimally.

\section{Sign-Definiteness Lemma with Multiple UnCertainties}

In this section we formally generalize Petersen's sign-definiteness lemma [14], [48], [49] to multiple complex valued uncertainties. The complex-valued version of this lemma for a single uncertainty is proved in [26]. The motivation for providing this novel extension is that the aforementioned versions of the lemma are inapplicable to solve the problems that we have formulated in Section II in this paper.

Lemma 1: Given matrices $\boldsymbol{A}$ and $\left\{\boldsymbol{P}_{i}, \boldsymbol{Q}_{i}\right\}_{i=1}^{N}$ with $\boldsymbol{A}=\boldsymbol{A}^{*}$, the semi-infinite Linear Matrix Inequality (LMI) of the form

$$
\boldsymbol{A} \succeq \sum_{i=1}^{N}\left(\boldsymbol{P}_{i}^{*} \boldsymbol{X}_{i} \boldsymbol{Q}_{i}+\boldsymbol{Q}_{i}^{*} \boldsymbol{X}_{i}^{*} \boldsymbol{P}_{i}\right), \quad \forall i, \boldsymbol{X}_{i}:\left\|\boldsymbol{X}_{i}\right\| \leq \varkappa_{i} ;
$$

holds if and only if there exist nonnegative real numbers $\epsilon_{1}, \cdots, \epsilon_{N}$ such that

$$
\left[\begin{array}{cccc}
\boldsymbol{A}-\sum_{i=1}^{N} \epsilon_{i} \boldsymbol{Q}_{i}^{*} \boldsymbol{Q}_{i} & -\varkappa_{1} \boldsymbol{P}_{1}^{*} & \cdots & -\varkappa_{N} \boldsymbol{P}_{N}^{*} \\
-\varkappa_{1} \boldsymbol{P}_{1} & \epsilon_{1} \boldsymbol{I} & \cdots & \mathbf{0} \\
\vdots & \vdots & \ddots & \vdots \\
-\varkappa_{N} \boldsymbol{P}_{N} & \mathbf{0} & \cdots & \epsilon_{N} \boldsymbol{I}
\end{array}\right] \succeq 0 .
$$

\footnotetext{
${ }^{2}$ Finding the global optimum even for a smooth non-convex non-linear problem (say, a problem with bilinear objective function or constraints, in our treatment, or generally a worst-case optimization problem which is a semi-infinite problem) is an NP-hard problem. See, e.g., [58], [59].
} 
The matrix norm in this lemma is the spectral norm. Later, it will be applied to models where $\boldsymbol{X}_{i}$ is a vector, and in that case the spectral norm is equal to the Frobenius norm.

Proof: ${ }^{3}$ The structure of the proof is to first use standard mathematical tools to transform (12), and then apply the $\mathcal{S}$-Procedure [50]. It is known that

$$
\boldsymbol{A} \succeq \sum_{i=1}^{N}\left(\boldsymbol{P}_{i}^{*} \boldsymbol{X}_{i} \boldsymbol{Q}_{i}+\boldsymbol{Q}_{i}^{*} \boldsymbol{X}_{i}^{*} \boldsymbol{P}_{i}\right), \quad \forall \boldsymbol{X}_{i}:\left\|\boldsymbol{X}_{i}\right\| \leq \varkappa_{i}, \quad i=1, \cdots, N ;
$$

holds if and only if for every $\boldsymbol{x}$ (based on the definition of a positive semi-definite matrix [50])

$$
\begin{aligned}
\boldsymbol{x}^{*} \boldsymbol{A} \boldsymbol{x} & \geq \max _{\left\{\mathfrak{c}_{i}\right\}_{i=1}^{N}} \sum_{i=1}^{N}\left(\boldsymbol{x}^{*} \boldsymbol{P}_{i}^{*} \boldsymbol{X}_{i} \boldsymbol{Q}_{i} \boldsymbol{x}+\boldsymbol{x}^{*} \boldsymbol{Q}_{i}^{*} \boldsymbol{X}_{i}^{*} \boldsymbol{P}_{i} \boldsymbol{x}\right) \\
& =2 \sum_{i=1}^{N} \varkappa_{i}\left\|\boldsymbol{P}_{i} \boldsymbol{x}\right\|\left\|\boldsymbol{Q}_{i} \boldsymbol{x}\right\|
\end{aligned}
$$

where

$$
\mathfrak{C}_{i}=\left\{\forall \boldsymbol{X}_{i}:\left\|\boldsymbol{X}_{i}\right\| \leq \varkappa_{i}\right\}, \quad i=1, \cdots, N
$$

Using the Cauchy-Schwarz inequality the above equation can be expressed as

$$
\boldsymbol{x}^{*} \boldsymbol{A} \boldsymbol{x}-2 \sum_{i=1}^{N} \varkappa_{i} \Re\left\{\boldsymbol{y}_{i}^{*} \boldsymbol{P}_{i} \boldsymbol{x}\right\} \geq 0, \quad \forall \boldsymbol{x}, \boldsymbol{y}_{i}:\left\|\boldsymbol{y}_{i}\right\| \leq\left\|\boldsymbol{Q}_{i} \boldsymbol{x}\right\|, \quad i=1, \cdots, N .
$$

Since $\left\|\boldsymbol{y}_{i}\right\| \leq\left\|\boldsymbol{Q}_{i} \boldsymbol{x}\right\|$ is equivalent to $\boldsymbol{x}^{*} \boldsymbol{Q}_{i}^{*} \boldsymbol{Q}_{i} \boldsymbol{x}-\boldsymbol{y}_{i}^{*} \boldsymbol{y}_{i} \succeq 0$, it is possible to express it in terms of a quadratic expression. By choosing $\boldsymbol{z}=\left[\boldsymbol{x}^{T}, \boldsymbol{y}_{1}^{T}, \cdots, \boldsymbol{y}_{N}^{T}\right]^{T}$, it is possible to write the above quadratic expression as $\boldsymbol{z}^{*} \mathcal{M}_{i} \boldsymbol{z} \geq 0$ where $\mathcal{M}_{i}$ is a block partitioned matrix, i.e., $\mathcal{M}_{i} \triangleq\left[\boldsymbol{M}^{[i]}{ }_{j, k}\right]_{(N+1) \times(N+1)}$ where

$$
\boldsymbol{M}_{j, k}^{[i]}= \begin{cases}\boldsymbol{Q}_{i}^{*} \boldsymbol{Q}_{i} & j=k=1, \\ -\boldsymbol{I} & j=k=i \neq 1, \\ \mathbf{0} & \text { otherwise }\end{cases}
$$

Using this notation it is possible to write (18) as another quadratic form, i.e.,

$$
\boldsymbol{x}^{*} \boldsymbol{A} \boldsymbol{x}-2 \sum_{i=1}^{N} \varkappa_{i} \Re\left\{\boldsymbol{y}_{i}^{*} \boldsymbol{P}_{i} \boldsymbol{x}\right\} \geq 0 \equiv \boldsymbol{z}^{*} \mathcal{M}_{0} \boldsymbol{z} \geq 0
$$

where $\mathcal{M}_{0}$ is a block partitioned matrix as well,

$$
\mathcal{M}_{0}=\left[\begin{array}{cccc}
\boldsymbol{A} & -\varkappa_{1} \boldsymbol{P}_{1}^{*} & \cdots & -\varkappa_{N} \boldsymbol{P}_{N}^{*} \\
-\varkappa_{1} \boldsymbol{P}_{1} & \mathbf{0} & \cdots & \mathbf{0} \\
\vdots & \vdots & \ddots & \vdots \\
-\varkappa_{N} \boldsymbol{P}_{N} & \mathbf{0} & \cdots & \mathbf{0}
\end{array}\right]
$$

\footnotetext{
${ }^{3}$ Our proof follows the footsteps of the proof of Proposition 2 of [26]. In our manuscript, equations (14) and (16) correspond to (102) and (103) of [26], except for that we have multiple uncertainties inside the summation. Unfortunately, (104) of [26] contains a typographical error and should read $\boldsymbol{x}^{*} \boldsymbol{A} \boldsymbol{x}-2 \rho \Re\left\{\boldsymbol{y}^{*} \boldsymbol{P} \boldsymbol{x}\right\} \geq 0$ instead; the corresponding equation in our paper is (18). In our proof, due to the requirements of our setup, we also need a change of variables to obtain a compact representation of the uncertainty sets. Hence, we provide a self-contained proof.
} 
Using this notation, it is possible to reformulate (18) as the following implication:

$$
\boldsymbol{z}^{*} \mathcal{M}_{i} \boldsymbol{z} \geq 0, \quad i=1, \cdots, N \quad \Rightarrow \quad \boldsymbol{z}^{*} \mathcal{M}_{0} \boldsymbol{z} \geq 0
$$

Using the general form of the $\mathcal{S}$-procedure [50] for quadratic functions and non-strict inequalities, (22) holds if there exists $\epsilon_{1}, \cdots, \epsilon_{N} \geq 0$ such that the LMI (13) holds and this completes the proof.

\section{Robust SOLUTION}

In this section we will present the robust solution to the problem formulations for the three different applications.

\section{A. Preliminaries}

Generally we use a two step solution. In the first step, we use the sign-definiteness lemma to convert the semi-infinite problem to a biconvex approximate version of that problem, and in the second step, we use an iterative algorithm to deal with the bi-convexity of that approximate problem. We first present a handy lemma which is helpful in formulating the performance measures, and then we give the solutions.

Lemma 2: For any set of zero-mean, independent and identically distributed random vectors with independent elements and individual covariances matrices $\mathrm{E}_{\boldsymbol{x}_{i}}\left[\boldsymbol{x}_{i} \boldsymbol{x}_{i}^{*}\right]=\sigma_{i}^{2} \boldsymbol{I}$ we have

$$
\mathrm{E}_{\left\{\boldsymbol{x}_{i}\right\}_{i}}\left[\left\|\sum_{i} \boldsymbol{A}_{i} \boldsymbol{x}_{i}\right\|_{2}^{2}\right]=\sum_{i} \sigma_{i}^{2}\left\|\boldsymbol{A}_{i}\right\|_{F}^{2}
$$

Proof: It is clear that

$$
\begin{aligned}
\mathrm{E}_{\left\{\boldsymbol{x}_{i}\right\}_{i}}\left[\left\|\sum_{i} \boldsymbol{A}_{i} \boldsymbol{x}_{i}\right\|_{2}^{2}\right] & =\sum_{i} \sum_{j} \mathrm{E}_{\boldsymbol{x}_{i}, \boldsymbol{x}_{j}}\left[\boldsymbol{x}_{i}^{*} \boldsymbol{A}_{i}^{*} \boldsymbol{A}_{j} \boldsymbol{x}_{j}\right] \\
& =\sum_{i} \sum_{j} \mathrm{E}_{\boldsymbol{x}_{i}, \boldsymbol{x}_{j}}\left[\operatorname{tr}\left[\boldsymbol{A}_{i}^{*} \boldsymbol{A}_{j} \boldsymbol{x}_{j} \boldsymbol{x}_{i}^{*}\right]\right] \\
& =\sum_{i} \sum_{j} \operatorname{tr}\left[\boldsymbol{A}_{i}^{*} \boldsymbol{A}_{j} \mathrm{E}_{\boldsymbol{x}_{i}, \boldsymbol{x}_{j}}\left[\boldsymbol{x}_{j} \boldsymbol{x}_{i}^{*}\right]\right] \\
& =\sum_{i} \sigma_{i}^{2} \operatorname{tr}\left[\boldsymbol{A}_{i}^{*} \boldsymbol{A}_{i}\right]
\end{aligned}
$$

which proves the lemma.

\section{B. Robust Solution to Application 1}

Using Lemma 2, $\operatorname{MSE}_{i}$ and $\operatorname{TxP}_{i}$ can be written:

$$
\begin{aligned}
\operatorname{TxP}_{i} & =\left\|\boldsymbol{P}_{i}\right\|_{F}^{2}, \quad \forall i, \\
\operatorname{MSE}_{i} & =\left\|\boldsymbol{D}_{i} \boldsymbol{H}_{i i} \boldsymbol{P}_{i}-\boldsymbol{I}_{S_{i}}\right\|_{F}^{2}+\sum_{\substack{j=1 \\
j \neq i}}^{I}\left\|\boldsymbol{D}_{i} \boldsymbol{H}_{j i} \boldsymbol{P}_{\boldsymbol{j}}\right\|_{F}^{2}+\sigma_{n_{i}}^{2}\left\|\boldsymbol{D}_{i}\right\|_{F}^{2}, \quad \forall i .
\end{aligned}
$$


Note that the MSE terms are norm-squared of matrix expressions that are bilinear in the design variables. Hence, the MSE constraints are not only semi-infinite with order $I$, but also non-convex. This is what makes the aforementioned problems hard to solve. Stressing on this observation, we outline our solution methodology as follows:

1) We first show that $\mathrm{MSE}_{i}$ can be represented as the norm-squared of a vector which is affine in the channel uncertainties. Then using Lemma 1, we find an equivalent SDP formulation for the problem at hand.

2) We then resort to an iterative algorithm based on the Alternating Convex Search (ACS) algorithm [52], to overcome the non-convexity of the problem.

In the following we will describe these steps in more detail.

1) Step 1: Using the identity $\|\boldsymbol{X}\|_{F}=\|\operatorname{vec}[\boldsymbol{X}]\|_{2}$ for any given matrix $\boldsymbol{X}$, we can rewrite $\operatorname{MSE}_{i}$ as the norm-squared of a vector:

$$
\mathrm{MSE}_{i}=\left\|\boldsymbol{m}_{i}\right\|_{2}^{2}
$$

where

$$
\boldsymbol{m}_{i} \triangleq\left[\begin{array}{c}
\operatorname{vec}\left[\boldsymbol{D}_{i} \boldsymbol{H}_{1 i} \boldsymbol{P}_{1}\right] \\
\vdots \\
\operatorname{vec}\left[\boldsymbol{D}_{i} \boldsymbol{H}_{I i} \boldsymbol{P}_{I}\right] \\
\sigma_{n_{i}}^{2} \operatorname{vec}\left[\boldsymbol{D}_{i}\right]
\end{array}\right]-\mathfrak{i}_{i} \in \mathbb{C}^{S_{i}\left(\sum_{j=1}^{I} S_{j}+R_{i}\right)}
$$

and

$$
\mathfrak{i}_{i}=\left[\begin{array}{c}
\mathbf{0}_{S_{i} \sum_{j=1}^{i-1} S_{j} \times 1} \\
\operatorname{vec}\left[\boldsymbol{I}_{S_{i}}\right] \\
\mathbf{0}_{S_{i}\left(\sum_{j=i+1}^{I} S_{j}+R_{i}\right) \times 1}
\end{array}\right] .
$$

Using the identity vec $[\boldsymbol{A B C}]=\left(\boldsymbol{C}^{T} \otimes \boldsymbol{A}\right) \operatorname{vec}[\boldsymbol{B}]$ for matrices of compatible dimensions, it is possible to rewrite $\boldsymbol{m}_{i}$ as an affine combination of uncertainty terms:

$$
\boldsymbol{m}_{i}=\tilde{\boldsymbol{m}}_{i}+\sum_{j=1}^{I} \boldsymbol{M}_{i j} \operatorname{vec}\left[\boldsymbol{\Delta}_{j i}\right],
$$

where

$$
\tilde{\boldsymbol{m}}_{i}=\left[\begin{array}{c}
\operatorname{vec}\left[\boldsymbol{D}_{i} \tilde{\boldsymbol{H}}_{1 i} \boldsymbol{P}_{1}\right] \\
\vdots \\
\operatorname{vec}\left[\boldsymbol{D}_{i} \tilde{\boldsymbol{H}}_{I i} \boldsymbol{P}_{I}\right] \\
\sigma_{n_{i}}^{2} \operatorname{vec}\left[\boldsymbol{D}_{i}\right]
\end{array}\right]-\mathfrak{i}_{i} \in \mathbb{C}^{S_{i}\left(\sum_{j=1}^{I} S_{j}+R_{i}\right)}
$$

and

$$
\boldsymbol{M}_{i j}=\left[\begin{array}{c}
\mathbf{0}_{\left(S_{i} \sum_{k=1}^{j-1} S_{k}\right) \times R_{i} T_{i}} \\
\boldsymbol{P}_{j}^{T} \otimes \boldsymbol{D}_{i} \\
\mathbf{0}_{\left(S_{i}\left(\sum_{k=j+1}^{I} S_{k}+R_{i}\right)\right) \times R_{i} T_{i}}
\end{array}\right] .
$$


We next use the Schur Complement Lemma [50] to recast the MSE constraint as a matrix inequality, and then we exploit the structure devised above together with Lemma 1. We know that the MSE constraint, i.e., $\left\|\boldsymbol{m}_{i}\right\|_{2}^{2} \leq \tau_{i}$, can be represented in terms of the following $\mathrm{LMI}^{4}$

$$
\left[\begin{array}{cc}
\tau_{i} & \boldsymbol{m}_{i}^{*} \\
\boldsymbol{m}_{i} & \boldsymbol{I}
\end{array}\right] \succeq 0
$$

By inserting the structure of the MSE into the above equation we have

$$
\left[\begin{array}{cc}
\tau_{i} & \tilde{\boldsymbol{m}}_{i}^{*} \\
\tilde{\boldsymbol{m}}_{i} & \boldsymbol{I}_{S_{i}\left(R_{i}+\sum_{j=1}^{I} S_{j}\right)}
\end{array}\right] \succeq-\sum_{j=1}^{I}\left[\begin{array}{cc}
0 & \left(\boldsymbol{M}_{i j} \operatorname{vec}\left[\boldsymbol{\Delta}_{j i}\right]\right)^{*} \\
\boldsymbol{M}_{i j} \operatorname{vec}\left[\boldsymbol{\Delta}_{j i}\right] & \mathbf{0}_{S_{i}\left(\sum_{j=1}^{I} S_{j}+R_{i}\right)}
\end{array}\right] .
$$

By appropriately choosing the the parameters of the sign-definiteness lemma as follows,

$$
\begin{aligned}
\boldsymbol{A}_{i} & =\left[\begin{array}{cc}
\tau_{i} & \tilde{\boldsymbol{m}}_{i}^{*} \\
\tilde{\boldsymbol{m}}_{i} & \boldsymbol{I}_{S_{i}\left(R_{i}+\sum_{j=1}^{I} S_{j}\right)}
\end{array}\right], \\
\boldsymbol{Q}_{i j} & =\left[\begin{array}{ll}
-1 & \boldsymbol{0}_{1 \times\left(S_{i}\left(R_{i}+\sum_{j=1}^{I} S_{j}\right)\right)}
\end{array}\right], \\
\boldsymbol{P}_{i j} & =\left[\begin{array}{ll}
\mathbf{0}_{R_{i} T_{j} \times 1} & \boldsymbol{M}_{i j}^{*}
\end{array}\right],
\end{aligned}
$$

we can recast the $\mathrm{MSE}_{i}$ constraint as the following matrix inequality:

$$
\left[\begin{array}{cccc}
\boldsymbol{A}_{i}-\sum_{j=1}^{I} \epsilon_{i j} \boldsymbol{Q}_{i j}^{*} \boldsymbol{Q}_{i j} & -\delta_{i 1} \boldsymbol{P}_{i 1}^{*} & \cdots & -\delta_{i I} \boldsymbol{P}_{i I}^{*} \\
-\delta_{i 1} \boldsymbol{P}_{i 1} & \epsilon_{i 1} \boldsymbol{I} & \cdots & \mathbf{0} \\
\vdots & \vdots & \ddots & \vdots \\
-\delta_{i I} \boldsymbol{P}_{i I} & \mathbf{0} & \cdots & \epsilon_{i I} \boldsymbol{I}
\end{array}\right] \succeq \mathbf{0}
$$

Note that the non-negativity of the slack variables follow from the preceding LMI. By assembling all components, the sum-MSE minimization problem can now be written:

$$
\begin{aligned}
\underset{\left\{\boldsymbol{P}_{i}, \boldsymbol{D}_{i}, \tau_{i} \geq 0, \epsilon_{i j}\right\}_{i, j=1}^{I}}{\operatorname{minimize}} & \sum_{i=1}^{I} \tau_{i} \\
\text { subject to } & \operatorname{TxP}_{i} \leq \gamma_{i}, \quad \forall i, \\
& \text { (35a) holds, } \quad \forall i
\end{aligned}
$$

This problem is not semi-infinite anymore, but it is biconvex (due to the underlying structure of $\boldsymbol{A}_{i}$ and $\left.\boldsymbol{P}_{i j}\right)$. Next we propose an iterative algorithm based on the ACS algorithm to solve it numerically.

2) Step 2: In what follows, we minimize the sum-MSE by first fixing a subset of the variables so that the problem at hand reduces to a convex one in the remaining variables (including the slack variables). We use interior point methods to solve the resulting convex problem. We then fix the remaining subset of variables and do the same thing to update the first subset of variables. This process continues until the desired accuracy is reached or until a certain number of iterations has been carried out. The algorithm is summarized as follows:

\footnotetext{
${ }^{4}$ The robust version of a SOCP is generally an SDP (which means the complexity class of a robust problem is increased relative to the original non-robust problem), see [60] and the references therein. The LMI-based formulation appears to be the most appropriate SDP, because it is convex, and unlike the vector/matrix lifting process [51] which usually needs the non-convex rank-1 constraint relaxation, the LMI-based formulation does not need any further assumptions or processing.
} 


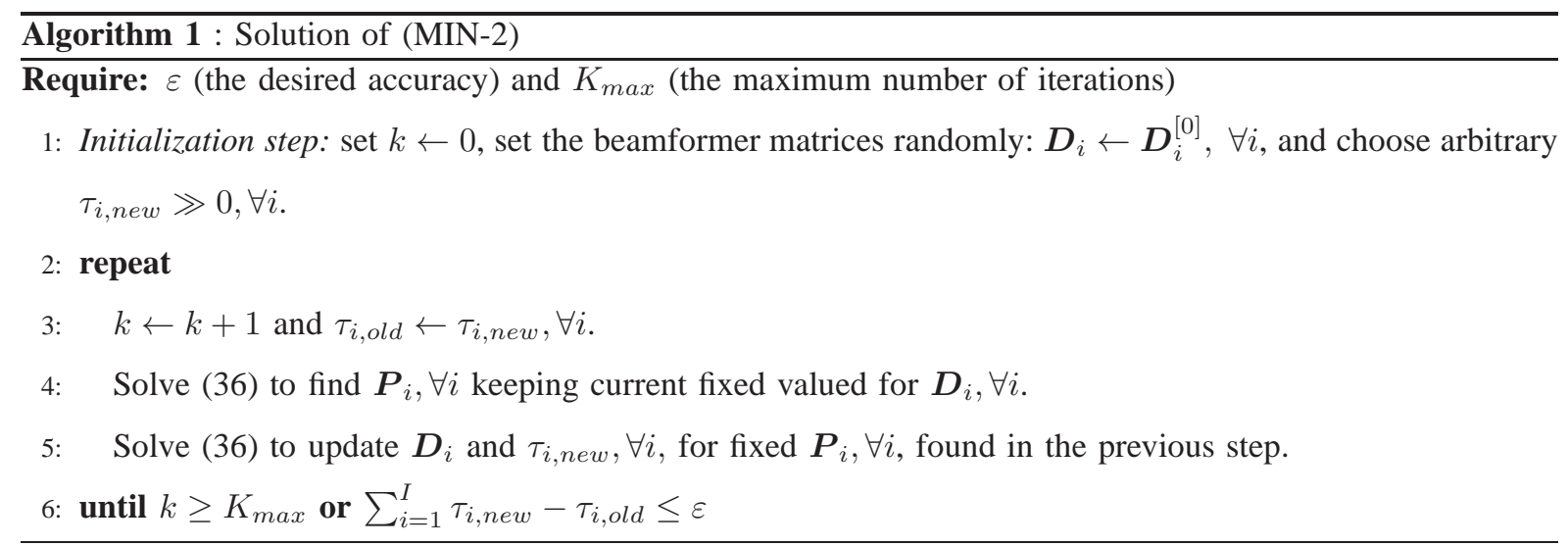

Clearly, the above algorithm will converge to a sub-optimal solution (provided that enough iterations are performed) as the objective function is bounded and non-increasing in each step. This is always the case for the ACS algorithm ${ }^{5}$ [52].

The general problem formulation proposed here reduces to the conventional perfect CSI case if we set the uncertainties zero. More precisely it is straightforward to show that the SDP formulation proposed herein would lead to the following SOCP when the uncertainty size is set to zero, and the slack variables are chosen to be arbitrary but positive:

$$
\begin{aligned}
\underset{\left\{\boldsymbol{P}_{i}, \boldsymbol{D}_{i}, \tau_{i} \geq 0\right\}_{i}}{\operatorname{minimize}} & \sum_{i=1}^{I} \tau_{i} \\
\text { subject to } & \operatorname{TxP}_{i} \leq \gamma_{i}, \quad \forall i \\
& \left\|\tilde{\boldsymbol{m}}_{i}\right\| \leq \tau_{i}, \quad \forall i
\end{aligned}
$$

We stress that the proposed algorithm is a centralized algorithm which means that there should be a dedicated station in the network which acquires all the relevant CSI, performs the steps of the algorithm and finally sends the transmitter and receiver parties back their required precoder or equalizer matrices. Although this mechanism may reduce the overall spectral efficiency of the system when taking into account the overhead, it appears to be indispensable. Since usually the receivers quantize the CSI before sending it to the central station, the linear equalizers designed in the central station have more uncertainty relative to the ones that could be designed by the receivers themselves [53].

\section{Robust Solution to Application 2}

In this section, we deal with the nonconvex problem (AFIRN-1) and convert it into a biconvex approximate problem, for which computationally efficient interior point methods exist. A similar

\footnotetext{
${ }^{5}$ The upper bound on the number of iterations $K_{\max }$, is included only to ensure that this algorithm always finishes with a fixed amount of time. In our numerical experiments, the algorithm using $K_{\max }=1000$ was never terminated by reaching the maximum number of iterations. If in some case, the convergence is very slow, we should change $K_{\max }$ accordingly to prevent premature termination of our algorithm. The optimum value for this parameter may be tuned experimentally for every application.
} 
procedure can be performed to simplify (AFIRN-2) and (AFIRN-3), details of this are omitted here. To do so, we employ a two stage process: first, we deal with the semi-infiniteness of the last two constraints of (AFIRN-1) using the generalized version of Petersen's lemma for complex valued matrices, and then we propose an iterative algorithm based on alternating convex search (ACS), to suboptimally solve the multilinear (nonconvex) problem. We start with the last constraint which, as noted in Remark 2, is multilinear in the design variables and threefold semi-infinite. The $\operatorname{TxP}_{r}$ constraint needs a similar procedure which is not repeated here. To deal with the MSE constraints, first using $\left\|\boldsymbol{A}_{i}\right\|_{F}=\left\|\operatorname{vec}\left[\boldsymbol{A}_{i}\right]\right\|_{2}$, the $\operatorname{MSE}_{i}, i=1,2$ is recast as follows:

$$
\operatorname{MSE}_{i}=\left\|\left[\begin{array}{c}
\operatorname{vec}\left[\boldsymbol{D}_{i}\left(\boldsymbol{G}_{i}+\boldsymbol{F}_{i} \boldsymbol{W} \boldsymbol{E}_{i}\right) \boldsymbol{P}_{i}-\boldsymbol{I}\right] \\
\left.\operatorname{vec}\left[\boldsymbol{D}_{i}\left[\left(\alpha_{i} \boldsymbol{H}_{i}+\boldsymbol{F}_{i} \boldsymbol{W} \boldsymbol{E}_{-i}\right) \boldsymbol{P}_{-i}-\left(1-\alpha_{i}\right) \boldsymbol{C}_{i}\right]\right]\right] \\
\sigma_{u} \operatorname{vec}\left[\boldsymbol{D}_{i} \boldsymbol{F}_{i} \boldsymbol{W}\right] \\
\sigma_{e_{i}} \operatorname{vec}\left[\boldsymbol{D}_{i}\right]
\end{array}\right]\right\|^{2} .
$$

After inserting (8) into the above equation, and neglecting higher order uncertainty terms ${ }^{6}$, it is possible to recast the $\operatorname{MSE}_{i}$ as $\operatorname{MSE}_{i}=\left\|\boldsymbol{\mu}_{i}\right\|^{2}$ where

$$
\boldsymbol{\mu}_{i} \triangleq \tilde{\boldsymbol{\mu}}_{i}+\sum_{\boldsymbol{X} \in \mathcal{U}_{i}} \boldsymbol{M}_{X} \operatorname{vec}\left[\boldsymbol{\Delta}_{X}\right]
$$

$\mathcal{U}_{i}=\left\{\boldsymbol{E}_{i,-i}, \boldsymbol{F}_{i}, \boldsymbol{G}_{i}, \boldsymbol{H}_{i}\right\}$, and

$$
\tilde{\boldsymbol{\mu}}_{i}=\left[\begin{array}{c}
\operatorname{vec}\left[\boldsymbol{D}_{i}\left(\tilde{\boldsymbol{G}}_{i}+\tilde{\boldsymbol{F}}_{i} \boldsymbol{W} \tilde{\boldsymbol{E}}_{i}\right) \boldsymbol{P}_{i}-\boldsymbol{I}\right] \\
\operatorname{vec}\left[\boldsymbol{D}_{i}\left[\left(\alpha_{i} \tilde{\boldsymbol{H}}_{i}+\tilde{\boldsymbol{F}}_{i} \boldsymbol{W} \tilde{\boldsymbol{E}}_{-i}\right) \boldsymbol{P}_{-i}-\left(1-\alpha_{i}\right) \boldsymbol{C}_{i}\right]\right] \\
\sigma_{u} \operatorname{vec}\left[\boldsymbol{D}_{i} \tilde{\boldsymbol{F}}_{i} \boldsymbol{W}\right] \\
\sigma_{e_{i}} \operatorname{vec}\left[\boldsymbol{D}_{i}\right]
\end{array}\right] \in \mathbb{C}^{S_{i}^{\prime}}
$$

${ }^{6} \boldsymbol{D}_{i} \boldsymbol{\Delta}_{F_{i}} \boldsymbol{W} \boldsymbol{\Delta}_{E_{i}} \boldsymbol{P}_{i}$ and $\boldsymbol{D}_{i} \boldsymbol{\Delta}_{F_{i}} \boldsymbol{W} \boldsymbol{\Delta}_{E_{-i}} \boldsymbol{P}_{i}$ have very small norms relative to the other terms and introduce a nonlinearity to the system which makes the problem mathematically intractable. 
and subsequently

$$
\begin{aligned}
\boldsymbol{M}_{G_{i}}= & {\left[\begin{array}{c}
\boldsymbol{P}_{i}^{T} \otimes \boldsymbol{D}_{i} \\
\mathbf{0}
\end{array}\right] \in \mathbb{C}^{m_{i}^{\prime} \times R_{i} T_{i}}, } \\
\boldsymbol{M}_{E_{i}}= & {\left[\begin{array}{c}
\boldsymbol{P}_{i}^{T} \otimes \boldsymbol{D}_{i} \tilde{\boldsymbol{F}}_{i} \boldsymbol{W} \\
\mathbf{0}
\end{array}\right] \in \mathbb{C}_{i}^{m_{i}^{\prime} \times r T_{i}}, } \\
\boldsymbol{M}_{H_{i}}= & {\left[\begin{array}{c}
\mathbf{0} \\
\alpha_{i} \boldsymbol{P}_{-i}^{T} \otimes \boldsymbol{D}_{i} \\
\mathbf{0}
\end{array}\right] \in \mathbb{C}^{m_{i}^{\prime} \times R_{i} T_{-i},} } \\
\boldsymbol{M}_{E_{-i}}= & {\left[\begin{array}{c}
\boldsymbol{0} \\
\boldsymbol{P}_{-i}^{T} \otimes \boldsymbol{D}_{i} \tilde{\boldsymbol{F}}_{i} \boldsymbol{W} \\
\mathbf{0}
\end{array}\right] \in \mathbb{C}^{m_{i}^{\prime} \times r T_{-i},} } \\
\boldsymbol{M}_{F_{i}}= & {\left[\begin{array}{c}
\left.\boldsymbol{W} \tilde{\boldsymbol{E}}_{i} \boldsymbol{P}_{i}\right)^{T} \otimes \boldsymbol{D}_{i} \\
\left(\boldsymbol{W} \tilde{\boldsymbol{E}}_{-i} \boldsymbol{P}_{-i}\right)^{T} \otimes \boldsymbol{D}_{i} \\
\sigma_{u} \boldsymbol{W}^{T} \otimes \boldsymbol{D}_{i} \\
\mathbf{0}
\end{array}\right] \in \mathbb{C}^{m_{i}^{\prime} \times R_{i} t}, }
\end{aligned}
$$

where $m_{i}^{\prime}=S_{i}\left(S_{i}+S_{-i}+r+R_{i}\right)$. Using the Schur complement lemma [46], the $\operatorname{MSE}_{i}$ constraint can be recast as the following LMI:

$$
\left[\begin{array}{cc}
\tau_{i} & \tilde{\boldsymbol{\mu}}_{i}^{*} \\
\tilde{\boldsymbol{\mu}}_{i} & \boldsymbol{I}
\end{array}\right] \succeq-\sum_{\boldsymbol{X} \in \mathcal{U}_{i}}\left[\begin{array}{cc}
0 & \left(\boldsymbol{M}_{\boldsymbol{X}} \operatorname{vec}\left[\boldsymbol{\Delta}_{\boldsymbol{X}}\right]\right)^{*} \\
\boldsymbol{M}_{\boldsymbol{X}} \operatorname{vec}\left[\boldsymbol{\Delta}_{\boldsymbol{X}}\right] & \mathbf{0}
\end{array}\right] .
$$

To proceed with this constraint, we will use the sign-definiteness lemma.

Using Lemma 1, and by appropriately choosing its parameters as follows (we have included $i$ to distinguish between two different constraints), we have:

$$
\begin{aligned}
\boldsymbol{A}_{i} & =\left[\begin{array}{cc}
\tau_{i} & \tilde{\boldsymbol{\mu}}_{i}^{*} \\
\tilde{\boldsymbol{\mu}}_{i} & \boldsymbol{I}
\end{array}\right] \in \mathbb{C}^{\left(1+m_{i}^{\prime}\right) \times\left(1+m_{i}^{\prime}\right)}, \\
\boldsymbol{Q}_{i 1} & =\boldsymbol{Q}_{i 2}=\boldsymbol{Q}_{i 3}=\boldsymbol{Q}_{i 4}=\boldsymbol{Q}_{i 5}=\left[\begin{array}{cc}
-1 & \mathbf{0}^{T}
\end{array}\right] \in \mathbb{C}^{1 \times\left(1+m_{i}^{\prime}\right)}, \\
\boldsymbol{P}_{i 1} & =\left[\begin{array}{ll}
\mathbf{0} & \boldsymbol{M}_{G_{i}}^{*}
\end{array}\right] \in \mathbb{C}^{R_{i} T_{i} \times\left(1+m_{i}^{\prime}\right)}, \boldsymbol{X}_{i 1}=\operatorname{vec}\left[\boldsymbol{\Delta}_{G_{i}}\right], \\
\boldsymbol{P}_{i 2} & =\left[\begin{array}{ll}
\mathbf{0} & \boldsymbol{M}_{E_{i}}^{*}
\end{array}\right] \in \mathbb{C}^{r T_{i} \times\left(1+m_{i}^{\prime}\right)}, \boldsymbol{X}_{i 2}=\operatorname{vec}\left[\boldsymbol{\Delta}_{E_{i}}\right], \\
\boldsymbol{P}_{i 3} & =\left[\begin{array}{ll}
\mathbf{0} & \boldsymbol{M}_{E_{-i}}^{*}
\end{array}\right] \in \mathbb{C}^{r T_{-i} \times\left(1+m_{i}^{\prime}\right)}, \boldsymbol{X}_{i 3}=\operatorname{vec}\left[\boldsymbol{\Delta}_{E_{-i}}\right], \\
\boldsymbol{P}_{i 4} & =\left[\begin{array}{ll}
\mathbf{0} & \boldsymbol{M}_{H_{i}}^{*}
\end{array}\right] \in \mathbb{C}^{R_{i} T_{-i} \times\left(1+m_{i}^{\prime}\right)}, \boldsymbol{X}_{i 4}=\operatorname{vec}\left[\boldsymbol{\Delta}_{H_{i}}\right], \\
\boldsymbol{P}_{i 5} & =\left[\begin{array}{ll}
\mathbf{0} & \boldsymbol{M}_{F_{i}}^{*}
\end{array}\right] \in \mathbb{C}^{t R_{i} \times\left(1+m_{i}^{\prime}\right)}, \boldsymbol{X}_{i 5}=\operatorname{vec}\left[\boldsymbol{\Delta}_{F_{i}}\right] .
\end{aligned}
$$

Now, it is possible to rewrite the $\operatorname{MSE}_{i}$ constraint as the following finite (single) LMIs:

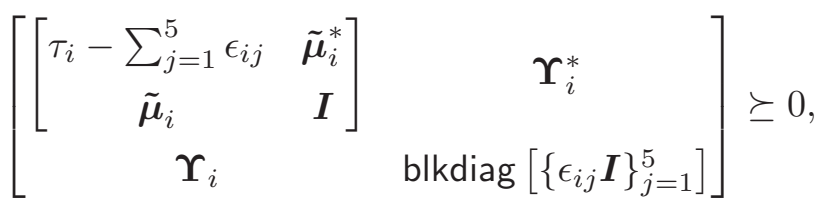


where

$$
\boldsymbol{\Upsilon}_{i}=-\left[\begin{array}{llllll}
\delta_{\boldsymbol{G}_{i}} \boldsymbol{P}_{i 1}^{T} & \delta_{\boldsymbol{E}_{i}} \boldsymbol{P}_{i 2}^{T} & \delta_{\boldsymbol{E}_{-i}} \boldsymbol{P}_{i 3}^{T} & \delta_{\boldsymbol{H}_{i}} \boldsymbol{P}_{i 4}^{T} & \delta_{\boldsymbol{F}_{i}} \boldsymbol{P}_{i 5}^{T}
\end{array}\right]^{T} .
$$

In this formulation, the non-negativity of the slack variables is automatically guaranteed, because the LMI in (42a) requires that the diagonal elements of the matrix on the left-hand side are positive. Hence $\tau_{i}$ also should be greater than or equal to the sum of $\epsilon_{i j}$ which itself is the sum of positive real numbers.

Using a similar procedure for the other semi-infinite constraint, i.e., the $\operatorname{TxP}_{r}$ constraint, it is possible to show that $\operatorname{TxP}_{r}=\|\boldsymbol{\pi}\|^{2}$, where

$$
\begin{gathered}
\boldsymbol{\pi}=\tilde{\boldsymbol{\pi}}+\sum_{i=1}^{2} \boldsymbol{P}_{E_{i}} \operatorname{vec}\left[\boldsymbol{\Delta}_{E_{i}}\right], \\
\tilde{\boldsymbol{\pi}}=\left[\begin{array}{c}
\operatorname{vec}\left[\boldsymbol{W} \tilde{\boldsymbol{E}}_{1} \boldsymbol{P}_{1}\right] \\
\operatorname{vec}\left[\boldsymbol{W} \tilde{\boldsymbol{E}}_{2} \boldsymbol{P}_{2}\right] \\
\sigma_{u} \operatorname{vec}[\boldsymbol{W}]
\end{array}\right] \in \mathbb{C}^{m^{\prime \prime}}, \\
\boldsymbol{P}_{E_{1}}=\left[\begin{array}{c}
\boldsymbol{P}_{1}^{T} \otimes \boldsymbol{W} \\
\mathbf{0}
\end{array}\right] \in \mathbb{C}^{m^{\prime \prime} \times r T_{1}}, \\
\boldsymbol{P}_{E_{2}}=\left[\begin{array}{c}
\mathbf{0} \\
\boldsymbol{P}_{2}^{T} \otimes \boldsymbol{W} \\
\mathbf{0}
\end{array}\right] \in \mathbb{C}^{m^{\prime \prime} \times r T_{2}},
\end{gathered}
$$

where $m^{\prime \prime}=t\left(S_{1}+S_{2}+r\right)$. Similarly, it is possible to replace this constraint with the following single LMI:

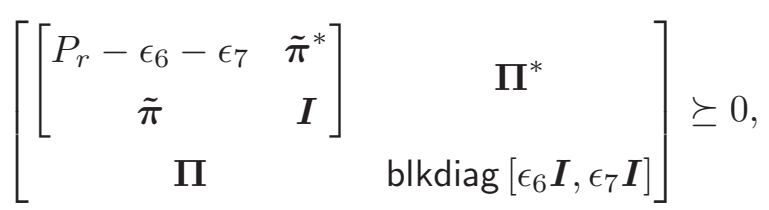

where

$$
\boldsymbol{\Pi}=\left[\begin{array}{lll}
\mathbf{0} & -\delta_{E_{1}} & \boldsymbol{P}_{E_{1}}^{*} \\
\mathbf{0} & -\delta_{E_{2}} & \boldsymbol{P}_{E_{2}}^{*}
\end{array}\right] \in \mathbb{C}^{r\left(T_{1}+T 2\right) \times\left(1+m^{\prime \prime}\right)} .
$$

Putting all these equivalent constraints together results in the following LMI which replaces (AFIRN-1):

$$
\begin{aligned}
\underset{\boldsymbol{P}_{i}, \boldsymbol{W}, \boldsymbol{D}_{i}, \epsilon_{1: 7} \geq 0, \tau_{i} \geq 0}{\operatorname{minimize}} & \tau_{1}+\tau_{2} \\
\text { subject to } & \left\|\boldsymbol{P}_{i}\right\|_{F}^{2} \leq P_{s_{i}}, \quad i=1,2 \\
& \text { (42a) holds, } \quad i=1,2 \\
& \text { (44a) holds, } \quad i=1,2
\end{aligned}
$$

This problem is no longer semi-infinite, but it is still non-convex. Due to the biconvex and multilinear structure of the elements of $\Pi$ and $\Upsilon_{i}$, we resort to an iterative algorithm inspired by the ACS method, see Algorithm 2. 


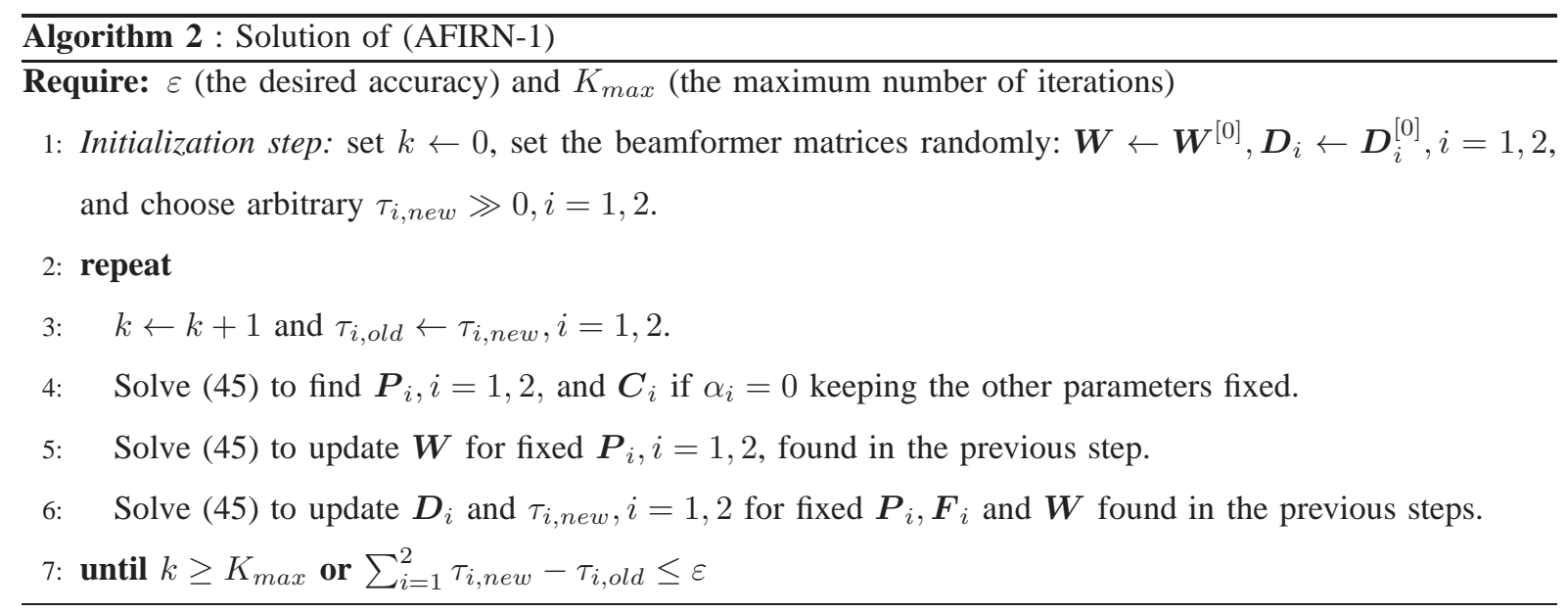

The convergence of the above algorithm (to at least a local optimum) is clear as the objective function decreases in each step and is bounded from below. Since the original problem is non-convex, we may obtain different solutions depending on how the algorithm is initialized.

It is straightforward to show that if $\delta_{G_{i}}=\delta_{H_{i}}=\delta_{E_{i}}=\delta_{F_{i}}=0$ the above problem reduces to the problem with full (perfect) CSI. In that case, (45) becomes a simple SOCP as follows:

$$
\begin{aligned}
\underset{\boldsymbol{P}_{i}, \boldsymbol{W}, \boldsymbol{D}_{i}, \boldsymbol{F}_{i}, \tau_{i}}{\operatorname{minimize}} & \tau_{1}+\tau_{2} \\
\text { subject to } & \left\|\boldsymbol{P}_{i}\right\|_{F}^{2} \leq P_{s_{i}}, \quad i=1,2, \\
& \|\tilde{\boldsymbol{\pi}}\|^{2} \leq P_{r}, \\
& \left\|\tilde{\boldsymbol{\mu}}_{i}\right\|^{2} \leq \tau_{i}, \quad i=1,2 .
\end{aligned}
$$

\section{Robust Solution to Application 3}

To solve the problem we need to find expressions for the MSE, $\operatorname{TxP}_{s}$ and the power $\operatorname{TxP}_{r_{i}}$. Using Lemma 2, we have:

$$
\begin{aligned}
& \mathrm{TxP}_{s}=\mathrm{E}_{\boldsymbol{s}}\left[\|\boldsymbol{P} \boldsymbol{s}\|^{2}\right]=\|\boldsymbol{P}\|_{F}^{2}, \\
& \operatorname{TxP}_{r_{i}}=\mathrm{E}_{\boldsymbol{s},\left\{\boldsymbol{e}_{i}\right\}_{i}}\left[\left\|\boldsymbol{W}_{i} \boldsymbol{G}_{i} \boldsymbol{P} \boldsymbol{s}+\boldsymbol{W}_{i} \boldsymbol{e}_{i}\right\|^{2}\right]=\left\|\boldsymbol{W}_{i} \boldsymbol{G}_{i} \boldsymbol{P}\right\|_{F}^{2}+\sigma_{e_{i}}^{2}\left\|\boldsymbol{W}_{i}\right\|_{F}^{2}, \\
& \operatorname{MSE}=\mathrm{E}_{\boldsymbol{s}, \boldsymbol{e}_{i}, \boldsymbol{n}}[\|\hat{\boldsymbol{s}}-\boldsymbol{s}\|] \\
& =\left\|\boldsymbol{D}\left(\sum_{i=1}^{L} \boldsymbol{H}_{i} \boldsymbol{W}_{i} \boldsymbol{G}_{i}\right) \boldsymbol{P}-\boldsymbol{I}_{S}\right\|_{F}^{2}+\sum_{i=1}^{L} \sigma_{e_{i}}^{2}\left\|\boldsymbol{D} \boldsymbol{H}_{i} \boldsymbol{W}_{i}\right\|_{F}^{2}+\sigma_{n}^{2}\|\boldsymbol{D}\|_{F}^{2} .
\end{aligned}
$$

To solve the design problem, we first show that it is possible to write the MSE as the norm of a vector which is an affine combination of the channel uncertainty terms. Then we use the sign-definiteness lemma to obtain a single matrix inequality instead of the original semi-infinite MSE constraint. Finally, we use an iterative algorithm based on the ACS method. 
The MSE can be written:

$$
\begin{aligned}
\mathrm{MSE} & =\|\boldsymbol{m}\|^{2} \\
& =\left\|\tilde{\boldsymbol{m}}+\sum_{i=1}^{L} \boldsymbol{M}_{G_{i}} \operatorname{vec}\left[\boldsymbol{\Lambda}_{i}\right]+\sum_{i=1}^{L} \boldsymbol{M}_{H_{i}} \operatorname{vec}[\boldsymbol{\Delta}]\right\|^{2},
\end{aligned}
$$

where

$$
\tilde{\boldsymbol{m}}=\left[\begin{array}{c}
\sum_{i=1}^{L} \operatorname{vec}\left[\boldsymbol{D} \tilde{\boldsymbol{H}}_{i} \boldsymbol{W}_{i} \tilde{\boldsymbol{G}}_{i} \boldsymbol{P}\right]-\operatorname{vec}\left[\boldsymbol{I}_{S}\right] \\
\sigma_{e_{1}} \operatorname{vec}\left[\boldsymbol{D} \tilde{\boldsymbol{H}}_{1} \boldsymbol{W}_{1}\right] \\
\vdots \\
\sigma_{e_{L}} \operatorname{vec}\left[\boldsymbol{D} \tilde{\boldsymbol{H}}_{L} \boldsymbol{W}_{L}\right] \\
\sigma_{n} \operatorname{vec}[\boldsymbol{D}]
\end{array}\right] \in \mathbb{C}^{S\left(S+\sum_{i=1}^{L} r_{i}+R\right)}
$$

and

$$
\begin{gathered}
\boldsymbol{M}_{G_{i}}=\left[\begin{array}{c}
\sum_{i=1}^{L} \boldsymbol{P}^{T} \otimes \boldsymbol{D} \tilde{\boldsymbol{H}}_{i} \boldsymbol{W}_{i} \\
\mathbf{0}_{S\left(R+\sum_{i=1}^{L} r_{i}\right) \times T r_{i}}
\end{array}\right], \\
\boldsymbol{M}_{H_{i}}=\left[\begin{array}{c}
\sum_{i=1}^{L}\left(\boldsymbol{W}_{i} \tilde{\boldsymbol{G}}_{i} \boldsymbol{P}\right)^{T} \otimes \boldsymbol{D} \\
\mathbf{0}_{S \sum_{j=1}^{i-1} r_{j} \times R t_{i}} \\
\boldsymbol{W}_{i}^{T} \otimes \boldsymbol{D} \\
\mathbf{0}_{S\left(R+\sum_{j=i+1}^{L} r_{j}\right) \times R t_{i}}^{L}
\end{array}\right] .
\end{gathered}
$$

By inserting these quantities into the LMI version of the MSE constraint, and choosing the signdefiniteness matrices as follows,

$$
\begin{aligned}
\boldsymbol{A} & =\left[\begin{array}{cc}
\tau & \tilde{\boldsymbol{m}}^{*} \\
\tilde{\boldsymbol{m}} & \boldsymbol{I}_{S\left(S+\sum_{i=1}^{L} r_{i}+R\right)}
\end{array}\right], \\
\boldsymbol{P}_{i} & = \begin{cases}{\left[\begin{array}{ll}
\mathbf{0}_{T r_{i} \times 1} & \boldsymbol{M}_{G_{i}}^{*}
\end{array}\right]} & i=1, \cdots, L \\
{\left[\begin{array}{ll}
\mathbf{0}_{R t_{i} \times 1} & \boldsymbol{M}_{H_{i}}^{*}
\end{array}\right]} & i=L+1, \cdots, 2 L\end{cases} \\
\boldsymbol{Q}_{i} & =\left[\begin{array}{ll}
-1 & \mathbf{0}_{1 \times S\left(S+\sum_{i=1}^{L} r_{i}+R\right)}
\end{array}\right] i=1, \cdots, 2 L,
\end{aligned}
$$

the MSE constraint of (MRN) takes on the following form:

where

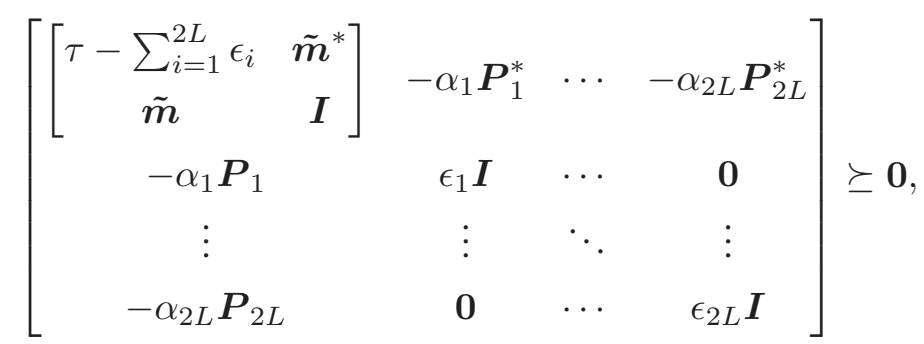

$$
\alpha_{i}= \begin{cases}\lambda_{i} & i=1, \cdots, L \\ \delta_{i} & i=I+1, \cdots, 2 L\end{cases}
$$


Similarly for the $\operatorname{TxP}_{r_{i}}$ constraint we can write

where

$$
\left[\begin{array}{ccc}
P_{r_{i}}-\eta_{i} & \tilde{\boldsymbol{p}}_{i}^{*} & \mathbf{0}_{1 \times r_{i} t_{i}} \\
\tilde{\boldsymbol{p}}_{i} & \boldsymbol{I}_{\left(S+r_{i}\right) t_{i}} & -\lambda_{i} \boldsymbol{\Psi}_{i} \\
\mathbf{0}_{r_{i} t_{i} \times 1} & -\lambda_{i} \boldsymbol{\Psi}_{i}^{*} & \eta_{i} \boldsymbol{I}_{r_{i} t_{i}}
\end{array}\right] \succeq \mathbf{0}
$$

and

$$
\tilde{\boldsymbol{p}}_{i}=\left[\begin{array}{c}
\operatorname{vec}\left[\boldsymbol{W}_{i} \tilde{\boldsymbol{G}}_{i} \boldsymbol{P}\right] \\
\sigma_{e_{i}} \operatorname{vec}\left[\boldsymbol{W}_{i}\right]
\end{array}\right],
$$

$$
\boldsymbol{\Psi}_{i}=\left[\begin{array}{c}
\boldsymbol{P}^{T} \otimes \boldsymbol{W}_{i} \\
\mathbf{0}_{r_{i} t_{i} \times T r_{i}}
\end{array}\right] .
$$

By putting all these components together the design problem becomes

$$
\begin{aligned}
\underset{\boldsymbol{P}, \boldsymbol{D},\left\{\boldsymbol{W}_{i}, \epsilon_{i}, \eta_{i}\right\}_{i}, \tau \geq 0}{\operatorname{minimize}} & \tau \\
\text { subject to } & \mathrm{TxP}_{s} \leq P_{s}, \\
& \text { (52a) holds, } \\
& \text { (53a) holds, } \forall i .
\end{aligned}
$$

Again we resort to an iterative ACS approach, summarized as follows:

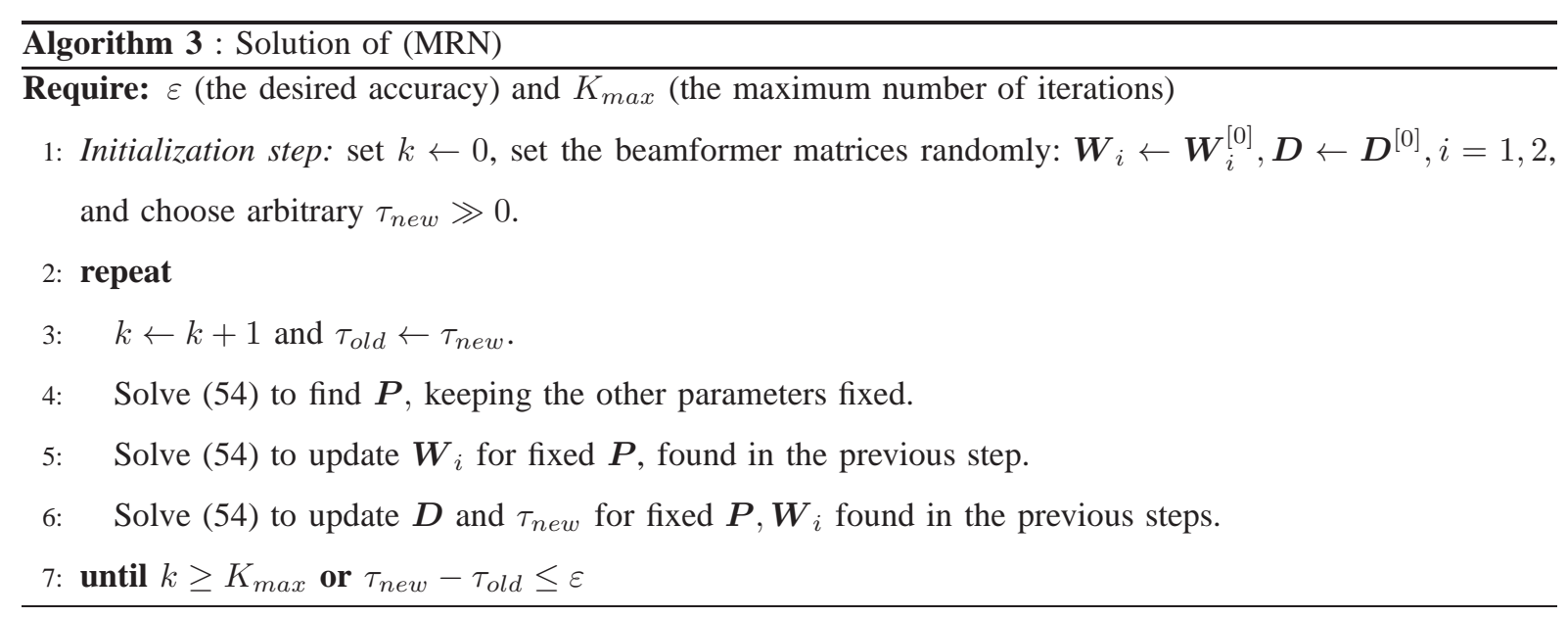

\section{E. Computational Complexity of the Proposed Algorithms}

In this sub-section we give some remarks on the computational complexity of the algorithms proposed earlier in this section. The proposed algorithms have two or three steps dealing with an SDP. So we focus on the complexity of each SDP. Consider a real-valued SDP problem in its standard form, i.e.,

$$
\begin{aligned}
\underset{\boldsymbol{x} \in \mathbb{R}^{n}}{\operatorname{minimize}} & \boldsymbol{c}^{T} \boldsymbol{x} \\
\text { subject to } & \boldsymbol{A}_{0}+\sum_{i=1}^{n} x_{i} \boldsymbol{A}_{i} \succeq \mathbf{0}
\end{aligned}
$$


where $\boldsymbol{A}_{i}$ are symmetric block-diagonal matrices with $K$ blocks of dimension $a_{k} \times a_{k}$. The number of operations required to solve this SDP is upper-bounded by [47]

$$
C\left(1+\sum_{k=1}^{K} a_{k}\right)^{1 / 2} n\left(n^{2}+n \sum_{k=1}^{K} a_{k}^{2}+\sum_{k=1}^{K} a_{k}^{3}\right),
$$

where $C$ is a constant that does not depend on the problem size. For example in Algorithm 1, to compute the optimal $\boldsymbol{P}_{i}$ in each iteration a SDP problem should be solved, for which the number of diagonal blocks $K$ is equal to $2 I$. For the first $I$ blocks, the dimension of the block is $a_{i}=$ $1+T_{i} S_{i} ; i=1, \cdots, I$ and for the next $I$ blocks the dimension of the blocks is equal to $a_{i}=$

$1+S_{i}\left(R_{i}+\sum_{j=1}^{I} S_{j}\right)+R_{i} \sum_{j=1}^{I} T_{j} ; i=1, \cdots, I$. In this problem we should determine $n=$ $I^{2}+I+2 \sum_{i=1}^{I} S_{i} T_{i}$ real variables. A similar analysis can be carried out for the next SDP in Algorithm 1, and also for the other algorithms.

In order to obtain some insight from (56), let us assume that the network has a large number of users $(I \gg 1)$, and that the number of transmit and receive antennas is larger than the number of independent streams of each link $\left(T_{i}, R_{i} \geq S_{i}\right)$. Also assume that we have a fully symmetric network, i.e., all the nodes have an equal number of antennas and streams $\left(S_{i}=S, T_{i}=T, R_{i}=R, \forall i\right)$. In this case, the required number of operations to get the precoder matrix is roughly

$$
K \times I^{5} \times S \times T^{4.5} \times R^{3.5}
$$

where $K$ is a constant. Hence in this example, doubling the number of data streams and transmit/receive antennas, increases the complexity by a factor in the order of $2^{9} \sim 500$.

\section{Simulation Results}

To illustrate the performance of the algorithm proposed in Section IV, we present here detailed numerical results for the interfering relay scenario in Section II-C. The simulation setup is as follows. The system is used to transfer $S_{1}=S_{2}=2$ streams of independent data symbols between the source and the destination. The numbers of transmit and receive antennas at the source, relay and destination nodes are equal to $T_{i}=R_{i}=t=r=4, i=1,2$. Both the source and relay power budgets are set to $P_{r}=P_{s_{1}}=P_{s_{2}}=1$. The convergence parameters of the algorithm are set to $K_{\max }=1000$, and $\varepsilon=10^{-4}$. We will present results for the case when $\alpha=1$, i.e., for a Gaussian IRC. The initial value of the relay precoder and the destination equalizer matrices are chosen at random. Due to the long computation time, we obtained the results by fixing the channel realization (at random) and averaging over 10000 perturbations. As for the distribution of the perturbations, we tried both Gaussian random matrices with a standard deviation proportional to the uncertainty size, and matrices on the sphere of uncertainty around the nominal value of the channel. These two choices yielded very similar results.

To demonstrate the convergence of the proposed algorithm, the empirical SMSE of the system as a function of the number of iterations is shown in Fig. 5. This figure shows the convergence of the algorithm for $\delta_{G_{i}}=\delta_{H_{i}}=\delta_{E_{i}}=\delta_{F_{i}}=0.01$ and different SNRs of $3 \mathrm{~dB}, 10 \mathrm{~dB}$, and $20 \mathrm{~dB}$ $\left(\sigma_{e_{i}}^{2}=\sigma_{u}^{2}=-3 \mathrm{~dB},-10 \mathrm{~dB},-20 \mathrm{~dB}\right)$. It is clear that at each iteration, the SMSE of the system decreases. After a few iterations the rate of change of the SMSE is small enough to terminate the 


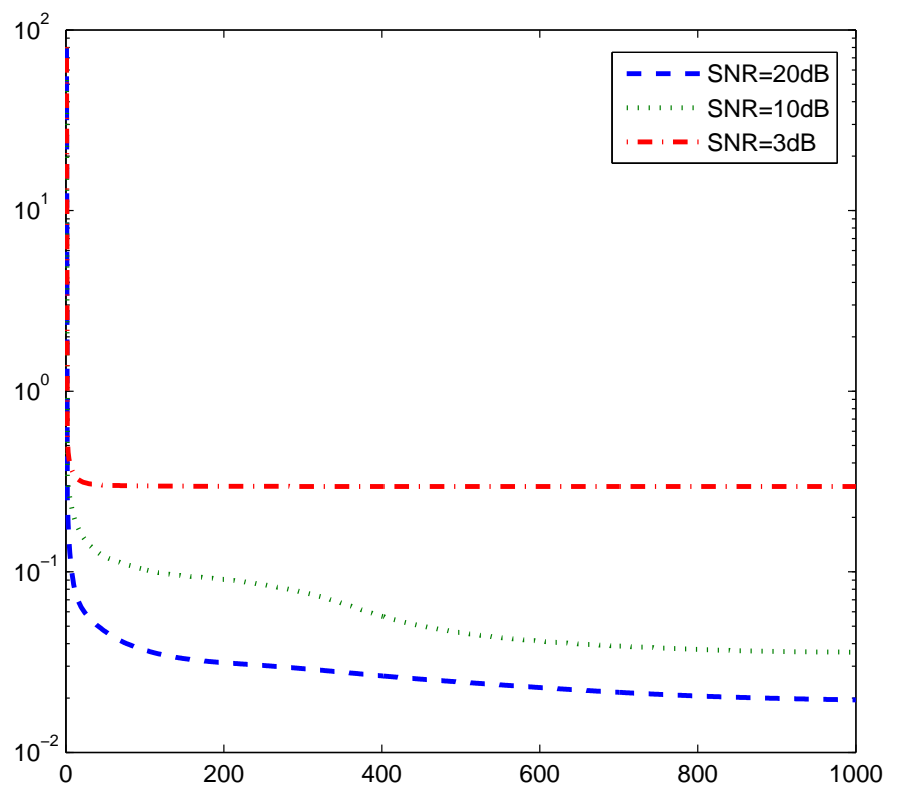

Fig. 5. Convergence of the SMSE minimization

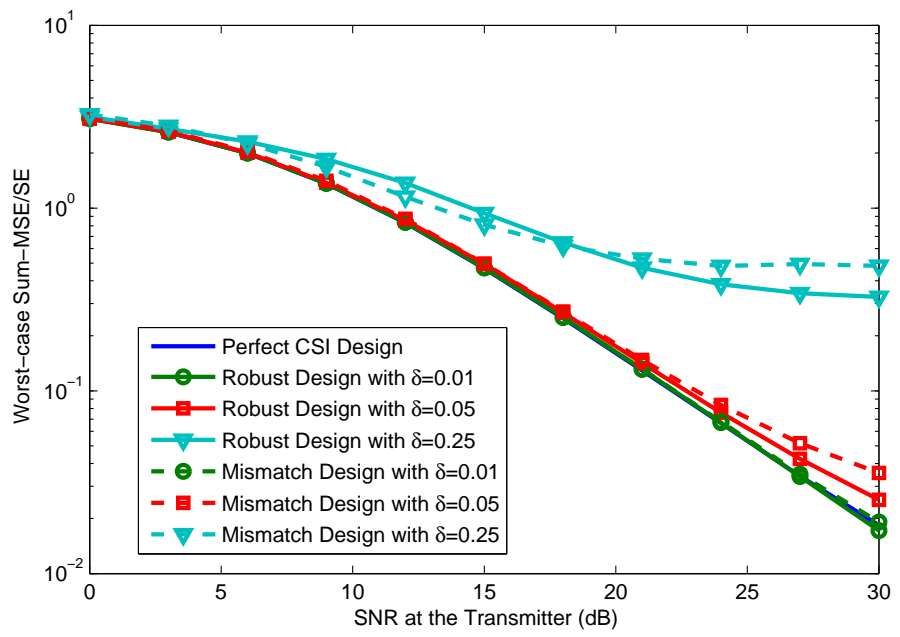

Fig. 6. System-wide Squared Error Norm for a GIFRC

algorithm. It is also clear that the algorithm converges to a (local) minimum. We also observe that for higher SNRs, the convergence rate decreases and the algorithms take more time to converge.

In Fig. 6 the worst-case squared error norm of the relay system is shown. When the noise power is small, the error is mostly dominated by the uncertainty terms rather than the noise terms, and for this reason the error is almost constant with respect to the noise power. In this regime, the error increases proportionally with the size of the uncertainty size. This is expected since $\boldsymbol{\mu}_{i}$ is a linear combination of the uncertainty matrices, and the uncertainty size is the norm of these matrices. However, for higher noise powers, the error is dominated by noise rather than by channel uncertainty, and therefore the error becomes a function only of the noise power but not of the uncertainty set size. The perfect 


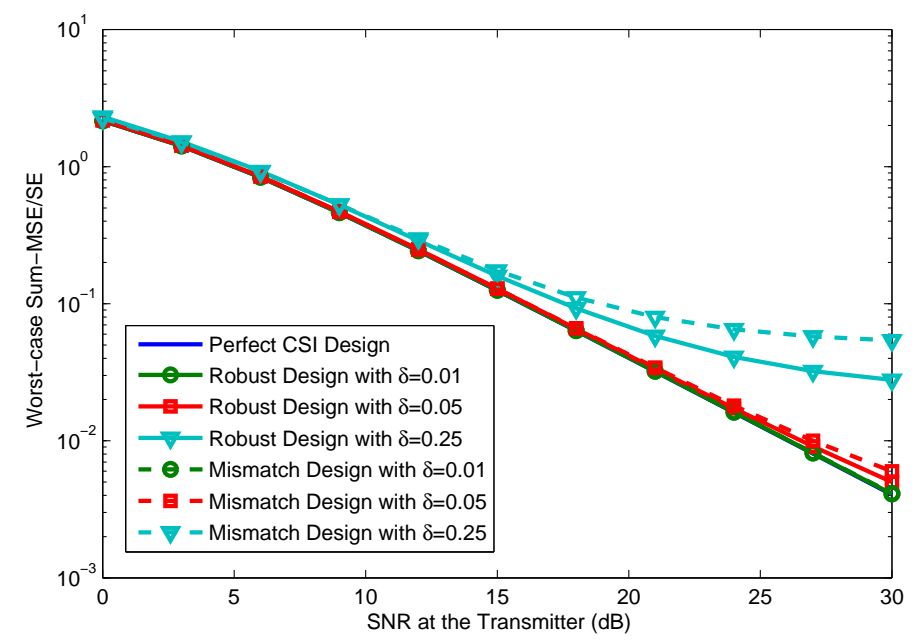

Fig. 7. System-wide Squared Error Norm for a TWRC

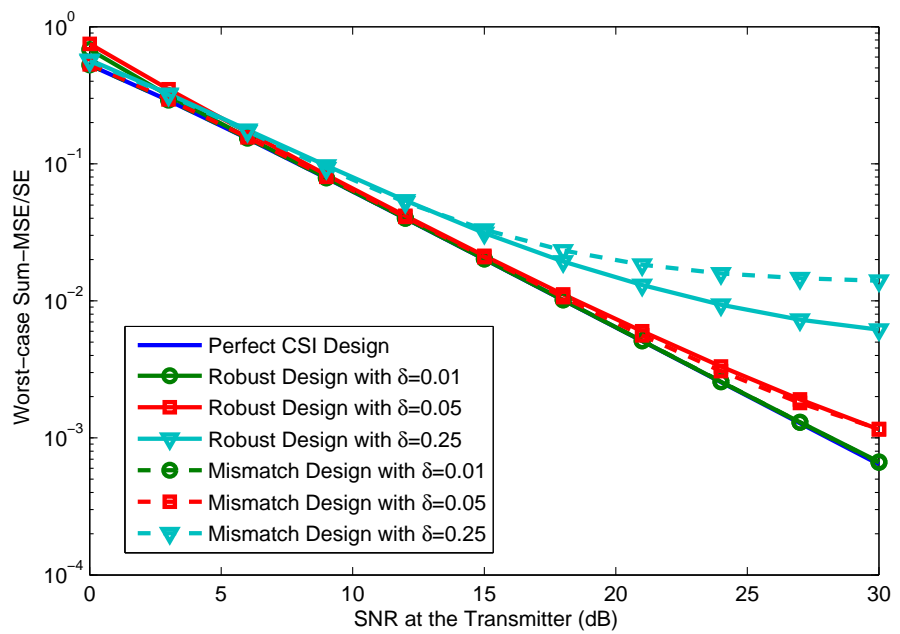

Fig. 8. System-wide Squared Error Norm for a Multi-Relay System with Two Relays

(full) CSI case outperforms the cases with CSI uncertainty, as expected. Note that the full CSI case is a special case of the partial CSI case with $\delta_{G_{i}}=\delta_{H_{i}}=\delta_{E_{i}}=\delta_{F_{i}} \triangleq \delta=0$. To better highlight the effectiveness of the proposed algorithms, we have included three extra curves (dashed curves) which show a "mismatched" scenario in which we assumed for the precoder design that the nominal CSI is the perfect CSI, while the actual CSI used when generating the data in the simulation had errors. It is clear that when the CSI errors are fairly small, the performance of the perfect CSI design is sensitive to errors in the high SNR regime, and in that case the squared error norm of the system is larger relative to our robust designs. On the other hand, when the CSI errors are very large, the performance of the baseline design is somewhat better than that of the robust design optimized to cope with the uncertainty, at low SNR. This is so because when the uncertainty is large enough, the actual CSI appears as completely random. For high SNR, the robust design generally outperforms the baseline design faced with mismatched CSI. Since in the mismatched design, the filters obtained from the nominal design may violate the transmit power constraint of the relay, we have normalized 


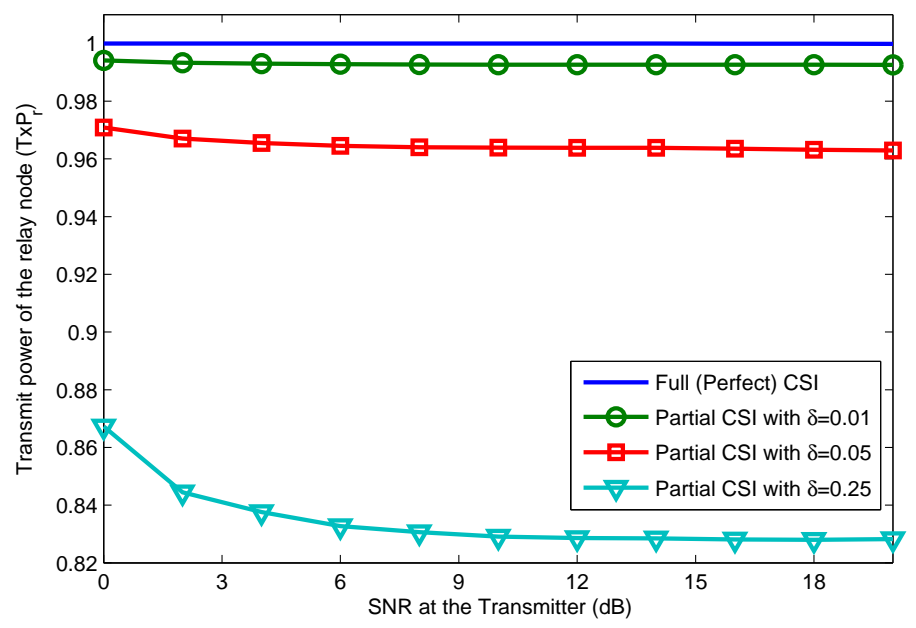

Fig. 9. Relay transmit power

the relay beamformer coefficients to prevent this violation, and then re-optimized the equalizer filters to be in line with this newly scaled beamformer. In Figs. 7 and 8 the system-wide MSE/Sum MSE is plotted for a TWRC and a multi-relay system with two relays. In both systems there is no direct link between the source node(s) and the destination(s). The number of transmit streams, transmit/receive antennas at the source/destination/relay are the same as in the first example of this section. Similar trends can be observed here as well.

In Fig. 9 the transmit power of the relay station is shown. Here the source transmit power constraints are always satisfied with equality. When there is no uncertainty, it is clear that by increasing the transmit power the MSE of the system decreases. Then the MSE decreases with TxP, which means that the TxP constraint is satisfied with equality. But when there is uncertainty in the CSI, there is no direct relation, because by increasing the TxP, we may increase the interfering power to other users as well. Hence, the relay transmit power constraint is not necessarily satisfied with equality. This is so because the optimal solution wants to prevent over-amplification of the relay noise. As can be seen, when the noise power increases, to maintain a minimum MSE, the transmit power of the relay station increases. It is clear that the transmit power is also a function of the channel uncertainty. When the uncertainty increases, the transmit power decreases. This is so because the MSE increases when the uncertainty increases, and to maintain the minimum possible MSE, the transmit power must be decreased. Intuitively, this can be understood by noting that since the nominal value of the channel is not a good estimate of the actual (true) CSI, and since the actual CSI could be very different from the nominal CSI, the algorithm does not rely on the available CSI estimate but instead transmits with less power over this channel.

\section{CONCLUSIONS}

The sign-definiteness lemma is a powerful tool that originates from control theory. In this paper we have presented a generalization of this lemma to complex-valued variables and multiple semi-infinite constraints. This generalization was used to solve three different MIMO transceiver optimization 
problems, namely that of interfering adhoc networks, general relay networks, and relay networks with multiple relays. We considered a (sum) MSE problem formulation in all three applications, and we have seen that the corresponding optimization problems are non-convex and semi-infinite. We used the sign-definiteness lemma to relax the semi-infiniteness of problems and we resorted to iterative algorithms based on ACS to devise a practical algorithm for solving the design problems. Simulation results show that when increasing the noise power and when increasing the size of the CSI uncertainty set, then the (sum) MSE increases. In the high SNR regime (where the noise power is low) the SMSE and the optimal transmit power are mainly affected by the CSI uncertainty set size. By contrast, at low SNR, the uncertainty size does not play an important role for the behavior of these quantities.

\section{REFERENCES}

[1] S. Skogestad, and I. Postlethwaite, Multivariable Feedback Control - Analysis and Design, John Wiley \& Sons, 1996.

[2] K. Zhou, Essentials of robust control, Prentice Hall, 1997.

[3] M. Bengtsson and B. Ottersten, "Optimal downlink beamforming using semidefinite optimization," Proceedings of 37th Annual Allerton Conference on Communication, Control, and Computing, pp. 987-996, Sep. 1999.

[4] S. A. Vorobyov, A. B. Gershman and Z. -Q. Luo, "Robust adaptive beamforming using worst-case performance optimization: A solution to the signal mismatch problem," IEEE Transactions On Signal Processing, vol. 51, no. 2, pp. 313-324, 2003.

[5] S. A. Vorobyov, A. B. Gershman, Z. -Q. Luo and N. Ma, "Adaptive beamforming with joint robustness against mismatched signal steering vector and interference nonstationarity," IEEE Signal Processing Letters, vol. 11, no. 2, pp. 108-111, 2004.

[6] S. A. Vorobyov, H. Chen and A. B. Gershman, "On the relationship between robust minimum variance beamformers with probabilistic and worst-case distortionless response constraints," IEEE Transactions On Signal Processing, vol. 56, no. 11, pp. 5719-5724, 2008.

[7] V. Sharma, I. Wajid, A. B. Gershman, H. Chen and S. Lambotharan, "Robust downlink beamforming using positive semidefinite covariance constraints," Proceedings of 2008 International ITG Workshop on Smart Antenna (WSA 2008), pp. 36-41, Feb. 2008.

[8] J. Li, P. Stoica, and Z. Wang “On robust Capon beamforming and diagonal loading," IEEE Trans. Signal Processing, vol. 51 , no. 7, pp. 1702-1715, Jul. 2003.

[9] A.B. Gershman, Z.-Q. Luo, and S. ShahbazPanahi, "Robust adaptive beamforming using worst-case performance optimization," chapter in the book Robust Adaptive Beamforming, P. Stoica and J. Li (Editors), John Wiley \& Sons, August 2005.

[10] S. ShahbazPanahi, A.B. Gershman, Z.-Q. Luo, and K.M. Wong, "Robust adaptive beamforming for general-rank signal models," IEEE Transactions on Signal Processing, vol. 51, pp. 2257-2269, Sept. 2003.

[11] A. Abdel-Samad, T.N. Davidson, and A.B. Gershman, "Robust transmit eigen beamforming based on imperfect channel state information," IEEE Trans. Signal Processing, vol. 54, no. 5, pp. 1596-1609, May 2006

[12] A. Mutapcic, S. -J. Kim and S. Boyd, "A tractable method for robust downlink beamforming in wireless communications," Proceedings of 2007 Asilomar Conference on Signals, Systems, and Computers (ACSSC 2007), pp. 1224-1228, Nov. 2007.

[13] S. -J. Kim, A. Magnani, A. Mutapcic, S. P. Boyd and Z. -Q. Luo, "Robust beamforming via worst-case SINR maximization" IEEE Transactions On Signal Processing, vol. 56, no. 4, pp. 1539-1574, 2008.

[14] I.R. Petersen, “A stabilization algorithm for a class of uncertain linear systems,” Systems \& Control Letters, no. 8, pp. $351-357,1987$.

[15] M.B. Shenouda, and T.N. Davidson, "Robust linear precoding for uncertain MISO broadcast channels," in Proc. ICASSP 2006, Toulouse, France, May 2006.

[16] M. Payaro, A. Pascual-Iserte, and M.A. Lagunas, "Robust power allocation designs for multiuser and multiantenna downlink communication systems through convex optimization,” IEEE J. Sel. Areas Commun., vol. 25, no. 7, pp. 1390-1401, Sep. 2007.

[17] N. Vucic and H. Boche, "Robust QoS-constrained optimization of downlink multiuser MISO systems," IEEE Trans. Signal Process., vol. 57, no. 2, pp. 714-725, Feb. 2009.

[18] M.B. Shenouda, and T.N. Davidson, "Convex conic formulations of robust downlink precoder design with quality of service constraints," IEEE J. Sel. Topics Signal Process., vol. 1, no. 4, pp. 714-724, Dec. 2007.

[19] E.A. Gharavol, Y.-C. Liang, and K. Mouthaan, "Robust downlink beamforming in multiuser MISO cognitive radio networks with imperfect channel state information," IEEE Trans. Vehicular Tech., vol. 59, No. 6, July 2010. 
[20] Y. Guo, and B.C. Levy, "Robust MSE equalizer design for MIMO communication systems in the presence of model uncertainties, IEEE Trans. Signal Process., vol. 54, no. 5, pp. 1840-1852, May 2006.

[21] N. Vucic, H. Boche, and S. Shi, "Robust transceiver optimization in downlink multiuser MIMO systems," IEEE Tran. Signal Process., vol. 57, no. 9, pp. 3576-3587, Sep. 2009.

[22] P. Ubaidulla, and A. Chockalingam, "Robust transceiver design for multiuser MIMO downlink," Proc. IEEE Global Telecommunications Conference, pp.1-5, Nov. 2008.

[23] J. Liu, and Z. Qiu, "Robust sum-MSE transceiver optimisation for multi-user non-regenerative MIMO relay downlink systems," Electronics Letters, vol. 47, no. 6, pp. 411-412, Mar. 2011.

[24] T.E. Bogale, B.K. Chalise, and L. Vandendorpe, "Robust transceiver optimization for downlink multiuser MIMO systems," IEEE Trans. Signal Processing, vol. 59, no. 1, pp. 446-453, Jan. 2011.

[25] E.A.Gharavol, Y.-C- Liang, and K. Mouthaan, "Robust linear transceiver design in MIMO adhoc cognitive radio networks with imperfect state information," IEEE Trans. Wireless Comm., vol. 10, No. 5, May 2011.

[26] Y.C. Eldar, A. Ben-Tal, and A. Nemirovski, "Robust mean-squared error estimation in the presence of model uncertainties," IEEE Tran. Signal Processing, vol. 53, no. 1, Jan. 2005.

[27] F. Gao, R. Zhang, and Y.-C. Liang, "Optimal channel estimation and training design for two-way relay networks," IEEE Trans. Communications, vol. 57, no. 10, pp. 3024-3033, Oct. 2009.

[28] —, "Channel estimation for OFDM modulated two-way relay networks," IEEE Trans. Signal Processing, vol. 57, no. 11, pp. 4443-4455, Nov. 2009.

[29] B. Jiang, F. Gao, X. Gao, and A. Nallanathan, "Channel estimation and training design for two-way relay networks with power allocation,” IEEE Trans. Wireless Communications, vol. 9, no. 6, pp. 2022-2032, Jun. 2010.

[30] T. Cui, F. Gao, T. Ho, and A. Nallanathan, "Distributed space-time coding for two-way wireless relay networks," IEEE Trans. Signal Processing, vol. 57, no. 2, pp. 658-671, Feb. 2009.

[31] V. Havary-Nassab, S. Shahbazpanahi, and A. Grami, "Optimal distributed beamforming for two-way relay networks," IEEE Trans. Signal Processing, vol.58, no.3, pp.1238-1250, Mar. 2010.

[32] J. Joung, and A.H. Sayed, "Multiuser two-way amplify-and-forward relay processing and power control methods for beamforming systems," IEEE Trans. on Signal Processing, vol.58, no.3, pp.1833-1846, Mar. 2010.

[33] I. Krikidis, and J.S Thompson, "MIMO two-way relay channel with superposition coding and imperfect channel estimation," Proc. IEEE GLOBECOM Workshops pp. 84-88, Dec. 2010.

[34] A.Y. Panah, and R.W. Heath, "MIMO two-way amplify-and-forward relaying with imperfect receiver CSI," IEEE. Trans. Vehicular Technology, vol. 59, no. 9, pp. 4377-4387, Nov. 2010.

[35] — "Sum-rate of MIMO two-way relaying with imperfect CSI," Proc. IEEE Conf. Acoustics Speech and Signal Processing (ICASSP), pp. 3418-3421, Mar. 2010.

[36] Y. Tian, and A. Yener, "The Gaussian interference relay channel: improved achievable rates and sum rate upper bounds using a potent relay," IEEE Trans. Information Theory, vol. 57, no. 5, pp. 2865-2879, May 2011.

[37] S. Sridharan, S. Vishwanath, S.A. Jafar, and S. Shamai, "On the capacity of cognitive relay assisted Gaussian interference channel," Proc. IEEE Information Theory Symp., pp. 549-553, Jul. 2008.

[38] I. Maric, R. Dabora, and A.J. Goldsmith, "An outer bound for the Gaussian interference channel with a relay," Proc. IEEE Information Theory Workshop, pp. 569-573, Oct. 2009.

[39] O. Simeone, O. Sahin, and E. Erkip, "Interference channel aided by an infrastructure relay," Proc. Information Theory Symp., pp. 2023-2027, Jun. 2009.

[40] Y. Shi, J.H. Wang, W.L. Huang, and K. Ben Letaief, "Power allocation in Gaussian interference relay channels via game theory," Proc. IEEE Global Telecommunications Conf., pp. 1-5, Nov. 2008.

[41] Y. Shi, J. Wang, K. Letaief, and R. Mallik, "A game-theoretic approach for distributed power control in interference relay channels," IEEE Trans. Wireless Communications, vol. 8, no. 6, pp. 3151-3161, Jun. 2009.

[42] P. Razaghi, and W. Yu "Universal relaying for the interference channel," Proc. Information Theory and Applications Workshop, pp. 1-6, Jan. 2010

[43] E.G. Larsson, E.A. Jorswieck, J. Lindblom, and R. Mochaourab, "Game theory and the flat-fading Gaussian interference channel: analyzing resource conflicts in wireless networks," IEEE Signal Processing Magazine, vol. 26, no. 5, pp. 18-27, Sept. 2009.

[44] F. Roemer, and M. Haardt, "Algebraic norm-maximizing (ANOMAX) transmit strategy for two-way relaying with MIMO amplify and forward relays," IEEE Signal Processing Letters, vol. 16, pp. 909-912, Oct. 2009. 
[45] J. Li, F. Roemer, and M. Haardt, "Efficient Relay Sharing (EReSh) between multiple operators in amplify-and-forward relaying systems," Proc. IEEE 4th Int. Workshop on Computational Advances in Multi-Sensor Adaptive Processing (CAMSAP 2011), pp. 249-252, Dec. 2011.

[46] K. B. Petersen and M. S. Pedersen, The Matrix Cookbook, Technical University of Denmark, Oct. 2008.

[47] A. Ben-Tal, and A. Nemirovski, Lectures on Modern Convex Optimization: Analysis, Algorithms, and Engineering Applications, ser. MPS-SIAM Series on Optimization, 2001.

[48] W.J. Mao, and J. Chu, "Quadratic stability and stabilization of dynamic interval systems," IEEE Trans. Automatic Control, vol. 48, no. 6, Jun. 2003.

[49] M.V. Khlebnikov, and P.S. Shcherbakov, "Petersen's lemma on matrix uncertainty and its generalizations," Automation and Remote Control, vol. 69, no. 11, pp. 1932-1945, 2008.

[50] S. Boyd, L. El Ghaoui, E. Ferron, and V. Balakrishnan, Linear Matrix Inequalities in System and Control Theory, Philadelphia, PA: SIAM, 1994.

[51] Z.-Q. Luo, W.-K. Ma, A.M.-C. So, Y. Ye, and S. Zhang "Semidefinite relaxation of quadratic optimization problems," IEEE Signal Processing Mag., vol. 27, no. 3, pp. 20-34, May 2010.

[52] J. Gorski, F. Pfeuffer, and K. Klamroth, "Biconvex sets and optimization with biconvex functions - a survey and extensions," Mathematical Methods of Operations Research, vol. 66, no. 3., pp. 373-408, Dec. 2007.

[53] M. Joham, P.M. Castro, L. Castedo, and W. Utschick, "Robust precoding with Bayesian error modeling for limited feedback MU-MISO systems," IEEE Trans. Signal Processing, vol. 48, no. 9, pp. 4954-4960, Sept. 2010.

[54] N. Farvardin, "A study of vector quantization for noisy channels," IEEE Trans. Information Theory vol. 36 , no. 4, pp. 799-809, 1990.

[55] M. Gastpar, B. Rimoldi, and M. Vetterli, "To code, or not to code: lossy source channel communication revisited," IEEE Trans. Information Theory, vol. 49, no. 5, pp. 1147-1158, May 2003.

[56] D. Persson, J. Kron, M. Skoglund, and E.G. Larsson, "Joint source-channel coding for the MIMO broadcast channel," IEEE Trans. Signal Processing, vol. 60, no. 4, pp. 2085-2090, Apr. 2012.

[57] A. Lozano, and N. Jindal, "Transmit diversity vs. spatial multiplexing in modern MIMO systems," IEEE Trans. Wireless Communications, vol. 9, no. 1, pp. 186-197, Jan. 2010.

[58] K.G. Murty, and S.N. Kabadi, "Some NP-Complete problems in quadratic and nonlinear programming," Mathematical Programming, no. 39, pp. 117-129, 1987.

[59] I.G. Akrotiriankis, and C.A. Floudas, "A new class of improved convex under-estimators for twice continuously differentiable constrained NLPs," Journal of Global Optimization, no. 20, pp. 367-390, 2004.

[60] D. Bertsimas, and M. Sim, "Tractable approximations to robust conic optimization problems," Mathematical Programming, vol. 1, no. 107, pp. 5-36, 2006.

[61] E.A. Gharavol, and E.G. Larsson, "Robust joint optimization of MIMO two-way relay channels with imperfect CSI," Proc. Allerton Conf. on Communication, Control, and Computing, pp. 1657 - 1664, Sept. 2011.

[62] E.A. Gharavol, and E.G. Larsson, "Robust joint optimization of non-regenerative MIMO relay channels with imperfect CSI", Proc. of the 45th Annual Asilomar Conf. Signals, Systems, and Computers, 2011.

[63] E.A. Gharavol, and E.G. Larsson, "Robust joint optimization of MIMO interfering relay channels with imperfect CSI," Proc. 4th IEEE International Workshop on Computational Advances in Multi-Sensor Adaptive Processing, pp. 209-212, Dec. 2011. 